\title{
New Private Types for the Cubic-Quartic Optical Solitons in Birefringent Fibers in Its Four Forms of Nonlinear Refractive Index
}

\section{Emad H.M. Zahran}

Benha University

Ahmet Bekir ( $\sim$ bekirahmet@gmail.com )

Eskisehir https://orcid.org/0000-0001-9394-4681

\section{Research Article}

Keywords: The cubic-quartic optical solitons in birefringent fibers, the extended simple equation method, the optical soliton solutions.

Posted Date: August 13th, 2021

DOl: https://doi.org/10.21203/rs.3.rs-762672/v1

License: (c) (i) This work is licensed under a Creative Commons Attribution 4.0 International License. Read Full License

Version of Record: A version of this preprint was published at Optical and Quantum Electronics on November 2nd, 2021. See the published version at https://doi.org/10.1007/s11082-021-03330-y. 


\title{
New private types for the cubic-quartic optical solitons in birefringent fibers in its four forms of nonlinear refractive index
}

\author{
Emad H.M. Zahran ${ }^{1}$, Ahmet Bekir ${ }^{2, *}$ \\ ${ }^{1}$ Departmentof Mathematical and Physical Engineering,BenhaUniversity,Faculty of \\ Engineering, Shubra, Egypt \\ ${ }^{2}$ Neighbourhood of Akcaglan, Imarli Street, Number: 28/4, 26030, Eskisehir, Turkey \\ *Corresponding author E-mail: bekirahmet@gmail.com ; e_h_zahran@hotmail.com
}

\begin{abstract}
From the point power of view of the extended simple equation method, multiple new private distinct types for the cubic-quartic optical soliton birefringent fibers with four forms nonlinear refractive index which have and play a vital effective effect in all modern telecommunications process have been extracted. The suggested method which continuously gives surprise accurate results for most nonlinear science problem has been implemented to extract multiple new accurate cubic-quartic soliton for the different forms of the NLSE which are, the cubicquartic in polarization-preserving fibers with the kerr-low nonlinearity, quadratic-cubic law nonlinearity, parabolic law nonlinearity and non-local law nonlinearity. Actual comparison between the achieved results and that demonstrated by other authors has been established.
\end{abstract}

Keywords: The cubic-quartic optical solitons in birefringent fibers, the extended simple equation method; the optical soliton solutions.

\section{1-Introduction}

Recently, it is important to study the transition of light through optical fibers which play principal rule in all wireless communication, modern telecommunications, audio waves and asocial media process. The birefringence is often quantified as the maximum difference between refractive indices exhibited by the material in general Crystals with non-cubic crystal structures are often birefringent, as are plastics under mechanical stress. In related subject Birefringence is used in many optical devices. Liquid-crystal displays, which are used utilized in medical diagnostics. In fact the double refraction phenomenon depends on Birefringence, because when a light ray incident upon a birefringent material it will split into two rays which have slightly various paths under the polarization property. In the literature this effect has been observed in calcite, a crystal having one of the strongest birefringence by the Danish scientist Rasmus Bartholin [1]. Moreover, Augustin-Jean Fresnel at 19th described the phenomenon in terms of polarization he can understand that the light can be considered as a wave with field components in transverse polarization. In addition the birefringence for which the optical property of a material having a refractive index that depends on the polarization and propagation direction of light has been discussed by $[2,3]$. Some studies have been demonstrated to investigate various forms of the NLSE which describes important nonlinear problems in different branches of sciences [4-14]. Recently, big number of authors discussed various forms of the cubic-quartic NLSE through their efforts to extract the soliton solutions 
via different techniques [15-25]. In the last few decays little studies through good efforts of some authors to various special forms of NLSE for the cubic-quartic of the birefringence for which the optical property of a material having a refractive index that depends on the polarization and propagation direction of light have been investigated through [26-28].

This article focused on applying the ESEM [8-9], [14] to achieve new types of optical solitons to cubic-quartic NLSE with its special forms which mentioned in the abstract. This article is organized as follow, the introduction section which gives short survey of the forms NLSE as well as the methods that used to solve it, in section two we will give description of the ESEM and its application to find the soliton solution of the suggested model. In section three the application the ESEM schema to extract optical soliton of the cubic-quartic for the Kerr-Low NLSE in polarization-preserving fibers. In section four, the application the ESEM schema to extract optical soliton of the cubic-quartic for the quadratic-low NLSE in polarizationpreserving fibers. In section five the application the ESEM schema to extract optical soliton of the cubic-quartic for the parabolic-low NLSE in polarization-preserving fibers. In section six the application the ESEM schema to extract optical soliton of the cubic-quartic for the Non-local-low NLSE in polarization-preserving fibers. In section seven brief conclusion of our work has been established.

\section{Description of the ESEM}

In overview, any nonlinear evolution equation can be written in the form,

$$
R\left(\psi, \psi_{x}, \psi_{t}, \psi_{x x}, \psi_{t t}, \ldots \ldots\right)=0 .
$$

Where $\mathrm{R}$ is a function of $\psi(x, t)$ and its partial derivatives that involves the highest order derivatives and nonlinear terms, according to the transformation $\psi(x, t)=\psi(\zeta), \zeta=x-C_{0} t$ equation (1) can be reduced to the following ODE:

$$
S\left(\psi, \psi^{\prime}, \psi^{\prime \prime}, \ldots \ldots \ldots . . .\right)=0
$$

Where $S$ is a function in $\psi(\zeta)$ and its total derivatives, while ${ }^{\prime}=\frac{d}{d \zeta}$

The constructed solution according to this method is:

$$
\psi(\zeta)=\sum_{i=-N}^{N} A_{i} \varphi^{i}(\zeta)
$$

Where the positive integer $N$ in Eq. (3) can be located by balancing the highest order derivative term and the nonlinear term, while the arbitrary constants $A_{i}$ could be calculated later, the function $\phi(\zeta)$ satisfies the following new ansatz equation

$$
\varphi^{\prime}(\zeta)=a_{0}+a_{1} \varphi+a_{2} \varphi^{2}
$$

Where $a_{0}, a_{1}$ and $a_{2}$ other arbitrary constants which admit these two cases;

(1) If $a_{1}=a_{3}=0$ it will transform to the Riccati equation [18-19], which has the following solutions;

$$
\begin{aligned}
& \varphi(\zeta)=\frac{\sqrt{a_{0} a_{2}}}{a_{2}} \tan \left(\sqrt{a_{0} a_{2}}\left(\zeta+\zeta_{0}\right), a_{0} a_{2} \mathrm{f} 0\right. \\
& \varphi(\zeta)=\frac{\sqrt{-a_{0} a_{2}}}{a_{2}} \tanh \left(\sqrt{-a_{0} a_{2}} \zeta-\frac{\rho \ln \zeta_{0}}{2}\right), a_{0} a_{2} \mathrm{p} 0, \zeta \mathrm{f} 0, \rho= \pm 1
\end{aligned}
$$


(2) If $a_{0}=a_{3}=0$, it will transform the Bernoulli equation [19], which has the following solutions;

$$
\begin{aligned}
& \varphi(\zeta)=\frac{a_{1} \operatorname{Exp}\left[a_{1}\left(\zeta+\zeta_{0}\right)\right]}{1-a_{2} \operatorname{Exp}\left[a_{1}\left(\zeta+\zeta_{0}\right)\right]}, a_{1} \mathrm{f} 0 \\
& \varphi(\zeta)=\frac{-a_{1} \operatorname{Exp}\left[a_{1}\left(\zeta+\zeta_{0}\right)\right]}{1+a_{2} \operatorname{Exp}\left[a_{1}\left(\zeta+\zeta_{0}\right)\right]}, a_{1} \mathrm{p} 0
\end{aligned}
$$

And the general solution to ansatz equation (4) is as follows:

$$
\begin{aligned}
& \varphi(\zeta)=-\frac{1}{a_{2}}\left(a_{1}-\sqrt{4 a_{1} a_{2}-a_{1}^{2}} \tan \left(\frac{\sqrt{4 a_{1} a_{2}-a_{1}^{2}}}{2}\left(\zeta+\zeta_{0}\right)\right), 4 a_{1} a_{2} \mathrm{f} a_{1}^{2}, a_{2} \mathrm{f} 0,\right. \\
& \varphi(\zeta)=\frac{1}{a_{2}}\left(a_{1}+\sqrt{4 a_{1} a_{2}-a_{1}^{2}} \tanh \left(\frac{\sqrt{4 a_{1} a_{2}-a_{1}^{2}}}{2}\left(\zeta+\zeta_{0}\right)\right), 4 a_{1} a_{2} \mathrm{f} a_{1}^{2}, a_{2} \mathrm{p} 0 .\right.
\end{aligned}
$$

Where $\zeta_{0}$ denotes to integration constancy.

Finally, via inserting equation (3) into equation (4) and equating the coefficients of different powers of $\varphi^{i}$ to zero, we get a system of algebraic equations, by solving it we obtain the values of the unknown variable mentioned in these equations. Moreover, inserting these achieved variables into equation (4) then the aimed solutions have been extracted.

\section{The Cubic-Quartic NLSE for the Kerr-Low nonlinearity using the ESEM}

In the framework of [27-28] the cubic-quartic for the Kerr-low NLSE in polarizationpreserving fibers can be proposed in the form,

$$
i Q_{t}+i a Q_{x x x}+b Q_{x x x x}+c\left|Q^{2 m}\right| Q=0
$$

Where $a, b$ and $c$ denote respectively to the 3 -th order dispersion index, the 4 -th order dispersion index, Kerr-Low refractive coefficient while $i=\sqrt{-1}$.

The cubic-quartic NLSE in birefringent fiber for Kerr-Low are,

$$
\begin{aligned}
& i u_{t}+i a_{1} u_{x x x}+b_{1} u_{x x x x}+\left[c_{1}|u|^{2}+d_{1}|v|^{2}\right] u=0 \\
& i v_{t}+i a_{2} v_{x x x}+b_{2} v_{x x x x}+\left[c_{2}|v|^{2}+d_{2}|u|^{2}\right] v=0
\end{aligned}
$$

In the framework of the ESEM the solution is,

$$
\begin{aligned}
& u(x, t)=\psi_{1}(\zeta) e^{i \eta_{1}(x, t)}, \zeta=k_{1} x+w_{1} t, \eta=q_{1} x+\delta_{1} t \\
& v(x, t)=\psi_{2}(\zeta) e^{i \eta_{2}(x, t)}, \zeta=k_{2} x+w_{2} t, \eta=q_{2} x+\delta_{2} t
\end{aligned}
$$

Hence,

$$
\begin{aligned}
& u_{t}=i \delta_{1} \psi_{1} e^{i \eta_{1}}+w_{1} \psi_{1}^{\prime} e^{i \eta_{1}} \\
& u_{x}=i q_{1} \psi_{1} e^{i \eta_{1}}+k_{1} \psi_{1}^{\prime} e^{i \eta_{1}} \\
& u_{x x}=-q_{1}^{2} \psi_{1} e^{i \eta_{1}}+2 i k_{1} q_{1} \psi_{1}^{\prime} e^{i \eta_{1}}+k_{1}^{2} \psi_{1}^{\prime \prime} e^{i \eta_{1}} \\
& u_{x x x}=-i q_{1}^{3} \psi_{1} e^{i \eta_{1}}-3 k_{1} q_{1}^{2} \psi_{1}^{\prime} e^{i \eta_{1}}+3 i q_{1} k_{1}^{2} \psi_{1}^{\prime \prime} e^{i \eta_{1}}+k_{1}^{3} \psi_{1}^{\prime \prime \prime} e^{i \eta_{1}}
\end{aligned}
$$




$$
\begin{aligned}
& u_{x x x x}=q_{1}^{4} \psi_{1} e^{i \eta_{1}}-4 i k_{1} q_{1}^{3} \psi_{1}^{\prime} e^{i \eta_{1}}-6 k_{1}^{2} q_{1}^{2} \psi_{1}^{\prime \prime} e^{i \eta_{1}}+4 i q_{1} k_{1}^{3} \psi_{1}^{\prime \prime \prime} e^{i \eta_{1}}+k_{1}^{4} \psi_{1}^{\prime \prime \prime \prime} e^{i \eta_{1}} \\
& \mid u^{2}=\psi_{1}^{2} \\
& v_{t}=i \delta_{2} \psi_{2} e^{i \eta_{2}}+w_{2} \psi_{2}^{\prime} e^{i \eta_{2}} \\
& v_{x}=i q_{2} \psi_{2} e^{i \eta_{2}}+k_{2} \psi_{2}^{\prime} e^{i \eta_{2}} \\
& v_{x x}=-q_{2}^{2} \psi_{2} e^{i \eta_{2}}+2 i k_{2} q_{2} \psi_{2}^{\prime} e^{i \eta_{2}}+k_{2}^{2} \psi_{2}^{\prime \prime} e^{i \eta_{2}} \\
& v_{x x x}=-i q_{2}^{3} \psi_{2} e^{i \eta_{2}}-3 k_{2} q_{2}^{2} \psi_{2}^{\prime} e^{i \eta_{2}}+3 i q_{2} k_{2}^{2} \psi_{2}^{\prime \prime} e^{i \eta_{2}}+k_{2}^{3} \psi_{2}^{\prime \prime \prime} e^{i \eta_{2}} \\
& v_{x x x x}=q_{2}^{4} \psi_{2} e^{i \eta_{2}}-4 i k_{2} q_{2}^{3} \psi_{1}^{\prime} e^{i \eta_{2}}-6 k_{2}^{2} q_{2}^{2} \psi_{2}^{\prime \prime} e^{i \eta_{2}}+4 i q_{2} k_{2}^{3} \psi_{2}^{\prime \prime \prime} e^{i \eta_{2}}+k_{2}^{4} \psi_{2}^{\prime \prime \prime \prime} e^{i \eta_{2}} \\
& \mid v^{2}=\psi_{2}^{2}
\end{aligned}
$$

Via inserting the relations (13) and (15-20) into the first part of Eq. (12) and the relations (14) and (21-26) into the second part of Eq. (12), the following real and imaginary parts must be emerged respectively,

$$
\begin{aligned}
& \operatorname{Re} \Rightarrow b_{j} k_{j}^{4} \psi_{j}^{\prime \prime \prime \prime}-\left(3 a_{j} q_{j} k_{j}^{2}+6 b_{j} q_{j}^{2} k_{j}^{2}\right) \psi_{j}^{\prime \prime}+\left(b_{j} q_{j}^{4}+6 a_{j} q_{j}^{3}-\delta_{j}\right) \psi_{j}+\left(c_{j}+d_{j}\right) \psi_{j}^{3}=0 . \\
& \operatorname{Im} \Rightarrow\left(a_{j} k_{j}^{3}+4 b_{j} q_{j} k_{j}^{3}\right) \psi_{j}^{\prime \prime \prime}-\left(3 k_{j} a_{j} q_{j}^{2}+4 k_{j} b_{j} q_{j}^{3}-w_{j}\right) \psi_{j}^{\prime}=0 .
\end{aligned}
$$

Equation (28) implies $a_{j}=-4 b_{j} q_{j}, w_{j}=3 k_{j} a_{j} q_{j}^{2}+4 k_{j} b_{j} q_{j}^{3}$, hence under this constrain Eq. (27) will be converted to,

$$
b_{j} k_{j}^{4} \psi_{j}^{\prime \prime \prime \prime}+6 b_{j} q_{j}^{2} k_{j}^{2} \psi_{j}^{\prime \prime}-\left(3 b_{j} q_{j}^{4}+\delta_{j}\right) \psi_{j}+\left(c_{j}+d_{j}\right) \psi_{j}^{3}=0 .
$$

Now, via integrating Eq. (29) with respect to $\zeta$ it becomes,

$$
b_{j} k_{j}^{4} \psi_{j}^{\prime \prime \prime}+6 b_{j} q_{j}^{2} k_{j}^{2} \psi_{j}^{\prime}-\frac{1}{2}\left(3 b_{j} q_{j}^{4}+\delta_{j}\right) \psi_{j}^{2}+\frac{1}{4}\left(c_{j}+d_{j}\right) \psi_{j}^{4}=0 .
$$

Equation (30) represents either the first or the second parts of equation (12) which are the same, for similarity we will implement the solution for the first part $j=1$ which is,

$$
b_{1} k_{1}^{4} \psi_{1}^{\prime \prime \prime}+6 b_{1} q_{1}^{2} k_{1}^{2} \psi_{1}^{\prime}-\frac{1}{2}\left(3 b_{1} q_{1}^{4}+\delta_{1}\right) \psi_{1}^{2}+\frac{1}{4}\left(c_{1}+d_{1}\right) \psi_{1}^{4}=0 .
$$

Via balancing $\psi_{1}^{\prime \prime \prime}, \psi_{1}^{4}$ at Eq. (31) leads to $4 N=N+3$ which implies that $N=1$, hence the solution is,

$$
\psi_{1}(\zeta)=\frac{A_{-1}}{\varphi}+A_{0}+A_{1} \varphi
$$

Where $\varphi^{\prime}=a_{0}+a_{1} \varphi+a_{2} \varphi^{2}+a_{3} \varphi^{3}$

Case 1: The first family, in which $a_{1}=a_{3}=0 \Rightarrow \varphi^{\prime}=a_{0}+a_{2} \varphi^{2}$, consequently

$$
\begin{aligned}
& \psi_{1}^{\prime}=-\frac{a_{0} A_{-1}}{\varphi^{2}}+A_{1} a_{0}+A_{1} a_{2} \varphi^{2}-a_{2} A_{-1} \\
& \psi_{1}^{\prime \prime \prime}=\frac{-6 a_{0}^{3} A_{-1}}{\varphi^{4}}-\frac{8 a_{0}^{2} a_{2} A_{-1}}{\varphi^{2}}-2 A_{-1} a_{0} a_{2}^{2}+2 A_{1} a_{2} a_{0}^{2}+8 A_{1} a_{0} a_{2}^{2} \varphi^{2}+6 A_{1} a_{2}^{3} \varphi^{4}
\end{aligned}
$$

Via inserting $\psi_{1}^{\prime \prime \prime}, \psi_{1}^{\prime}, \psi_{1}^{2}$ and $\psi_{1}^{4}$ into equation (31), collecting and equating the coefficients of various powers of $\varphi^{i}$ to zero leads to system of equations, by solving it we get these results, 


$$
\begin{aligned}
& a_{0}=\frac{-\sqrt{3 A_{-1}} q_{1}}{2 k_{1} \sqrt{A}}, a_{2}=\frac{\sqrt{3 A_{1}} q_{1}}{2 k_{1} \sqrt{A_{-}}}, \delta_{1}=\frac{3\left(\frac{-6 \sqrt{3 A_{1}} b_{1} k_{1} q_{1}^{3}}{\sqrt{A_{-}}}-A_{1} b_{1} q_{1}^{4}\right)}{A}, c_{1}=\frac{-d_{1} A_{1}^{3}-\left(\frac{9 \sqrt{3} A_{1}^{\frac{3}{2}} b_{1} k_{1} q_{1}^{3}}{A_{-1}^{\frac{3}{2}}}\right)}{A_{1}^{3}}, A_{0}=0 \\
& \left.a_{0}=\frac{\sqrt{3 A_{-1}} q_{1}}{2 k_{1} \sqrt{A}}, a_{2}=\frac{-\sqrt{3 A} q_{1}}{2 k_{1} \sqrt{A_{-}}}, \delta_{1}=\frac{3\left(\frac{6 \sqrt{3 A} b_{1} k_{1} q_{1}^{3}}{\sqrt{A_{1}}}-A b_{1} q_{1}^{4}\right)}{A}, c_{1}=\frac{-d_{1} A_{1}^{3}+\left(\frac{9 \sqrt{3} A_{1}^{\frac{3}{2}} b_{1} k_{1} q_{1}^{3}}{\frac{3}{2}}\right)}{A_{-1}^{\frac{2}{2}}}\right), A_{0}=0 \\
& a_{0}=\frac{-3 q_{1}^{2}}{a_{2} k_{1}^{2}}, \delta_{1}=\frac{-3\left(2 a_{2} b_{1} k_{1}^{2} q_{1}^{2}+A_{1} b_{1} q_{1}^{2}\right)}{A_{1}}, c_{1}=\frac{-d_{1} A_{1}^{3}-24 a_{2}^{3} b_{1} q_{1}^{4}}{A_{1}^{3}}, A_{-1}=0, A_{0}=0 \\
& a_{0}=\frac{-3 q_{1}^{2}}{a_{2} k_{1}^{2}}, \delta_{1}=\frac{-3\left(-2 a_{2} b_{1} k_{1}^{2} q_{1}^{2}+A_{-1} b_{1} q_{1}^{2}\right)}{A_{-1}}, c_{1}=\frac{-d_{1} A_{-1}^{3}+24 a_{0}^{3} b_{1} q_{1}^{4}}{A_{-1}^{3}}, A_{1}=0, A_{0}=0
\end{aligned}
$$

These 4-differents results will achieve 4-various types of solutions, from which we will plot the extracted solutions for the first and the third results.

Firstly, for the first result Eq. (35):

$$
a_{0}=\frac{-\sqrt{3 A_{-1}} q_{1}}{2 k_{1} \sqrt{A_{1}}}, a_{2}=\frac{\sqrt{3 A_{1}} q_{1}}{2 k_{1} \sqrt{A_{-1}}}, \delta_{1}=\frac{3\left(\frac{-6 \sqrt{3 A_{1}} b_{1} k_{1} q_{1}^{3}}{\sqrt{A_{-1}}}-A_{1} b_{1} q_{1}^{4}\right)}{A_{1}}, c_{1}=\frac{-d_{1} A_{1}^{3}-\left(\frac{9 \sqrt{3} A_{1}^{\frac{3}{2}} b_{1} k_{1} q_{1}^{3}}{A_{-1}^{\frac{3}{2}}}\right)}{A_{1}^{3}}, A_{0}=0
$$

This result can be simplified to be,

$$
A_{-1}=1, A_{0}=0, A_{1}=1, a_{0}=-0.9, a_{2}=0.9, k_{1}=b_{1}=d_{1}=q_{1}=C_{0}=1, c_{1}=-16.6, \delta_{1}=-34.2, \zeta_{0}=2, \rho=-1
$$

From the point of view of the proposed method the solution is,

$$
\begin{aligned}
& \varphi(\zeta)=\frac{\sqrt{-a_{0} a_{2}}}{a_{2}} \tanh \left(\sqrt{-a_{0} a_{2} \zeta}-\frac{\rho \ln \zeta_{0}}{2}\right), a_{0} a_{2} \mathrm{p} 0, \zeta \mathrm{f} 0, \rho= \pm 1 \\
& \varphi(\zeta)=\tanh (0.9 \zeta+0.3) \\
& \varphi(\zeta)=\tanh (0.9 x-7.2 t+0.3) \\
& \psi_{1}(\zeta)=\frac{A_{-1}}{\varphi}+A_{0}+A_{1} \varphi \\
& \psi_{1}(\zeta)=\operatorname{coth}(0.9 x-7.2 t+0.3)+\tanh (0.9 x-7.2 t+0.3) \\
& u(x, t)=\psi_{1}(\zeta) e^{i \eta_{1}(x, t)}, \zeta=k_{1} x+w_{1} t, \eta_{1}=q_{1} x+\delta_{1} t \\
& u(x, t)=(\operatorname{coth}[0.9 x-7.2 t+0.3]+\tanh [0.9 x-7.2 t+0.3]) e^{i(x-34.2 t)} \\
& \operatorname{Re} u=(\operatorname{coth}[0.9 x-7.2 t+0.3]+\tanh [0.9 x-7.2 t+0.3]) \times \cos (x-34.2 t) \\
& \operatorname{Im} u=(\operatorname{coth}[0.9 x-7.2 t+0.3]+\tanh [0.9 x-7.2 t+0.3]) \times \sin (x-34.2 t)
\end{aligned}
$$



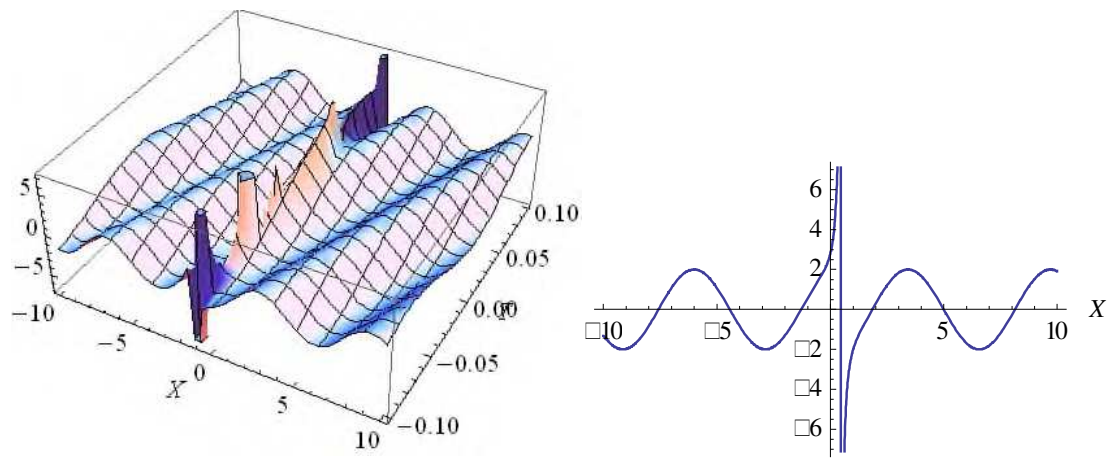

Fig.1 The cubic-quartic soliton of the Re. part of kerr-low Eq.(42) in 2D and 3D with values: $y_{1}=-8$ $A_{-1}=1, A_{0}=0, A_{1}=1, a_{0}=-0.9, a_{2}=0.9, k_{1}=b_{1}=d_{1}=q_{1}=C_{0}=1, c_{1}=-16.6, \delta_{1}=-34.2, \zeta_{0}=2, \rho=-1, t=0.1$ for $2 \mathrm{D}$
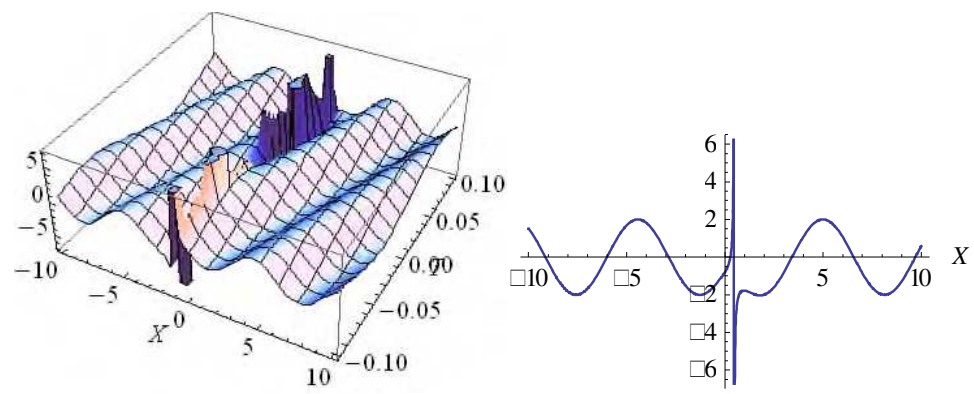

Fig.2 The cubic-quartic soliton of the Im. part of kerr-low Eq.(43) in 2D and 3D with values: $w_{1}=-8$ $A_{-1}=1, A_{0}=0, A_{1}=1, a_{0}=-0.9, a_{2}=0.9, k_{1}=b_{1}=d_{1}=q_{1}=C_{0}=1, c_{1}=-16.6, \delta_{1}=-34.2, \zeta_{0}=2, \rho=-1, t=0.1$ for $2 D$

Secondly, for the third result Eq, (36):

$$
a_{0}=\frac{-3 q_{1}^{2}}{a_{2} k_{1}^{3}}, \delta_{1}=\frac{-3 k_{1} b_{1} q_{1}^{2}\left(-12 a_{2}+A_{1} q_{1}^{2}\right)}{A_{1}}, c_{1}=\frac{-d_{1} A_{1}^{3}-24 a_{2}^{3} b_{1} q_{1}^{4}}{A_{1}^{3}}, A_{-1}=0, A_{0}=0
$$

This result can be simplified to be,

$$
A_{-1}=A_{0}=0, A_{1}=1, a_{0}=0.9+0.13 i, a_{2}=0.3+0.01 i, k_{1}=b_{1}=d_{1}=q_{1}=c_{1}=C_{0}=1, \delta_{1}=-4.8-0.6 i, \zeta_{0}=2, \rho=-1
$$

From the point of view of the proposed method the solution is,

$$
\begin{aligned}
& \varphi(\zeta)=\frac{\sqrt{a_{0} a_{2}}}{a_{2}} \tan \left(\sqrt{a_{0} a_{2}}\left(\zeta+\zeta_{0}\right), a_{0} a_{2} \mathrm{f} 0\right. \\
& \varphi(\zeta)=5.7 \tan [1.7 x-13.6 t+1.7] \\
& \psi(\zeta)=5.7 \tan [1.7 x-13.6 t+1.7] \\
& u(x, t)=5.7 \tan [1.7 x-13.6 t+1.7] \times e^{i[x-(4.8+0.6 i) t]} \\
& \operatorname{Re} u=5.7 \tan [1.7 x-13.6 t+1.7] \times e^{0.6 t} \times \cos (x-4.8 t) \\
& \operatorname{Im} u=5.7 \tan [1.7 x-13.6 t+1.7] \times e^{0.6 t} \times \sin (x-4.8 t)
\end{aligned}
$$




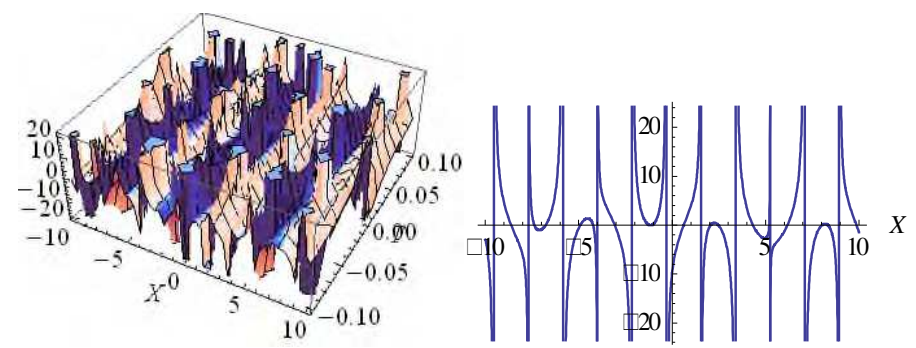

Fig.3 The cubic-quartic soliton of the Re. part of kerr-low Eq.(47) in 2D and 3D with values: $w_{1}=-8$ $A_{-1}=A_{0}=0, A_{1}=1,\left|a_{0}\right|=9.6,\left|a_{2}\right|=0.3, k_{1}=b_{1}=d_{1}=q_{1}=\zeta_{0}=C_{0}=1, c_{1}=-25, \delta_{1}=-4.8-0.6 i, t=0.1$ for $2 \mathrm{D}$

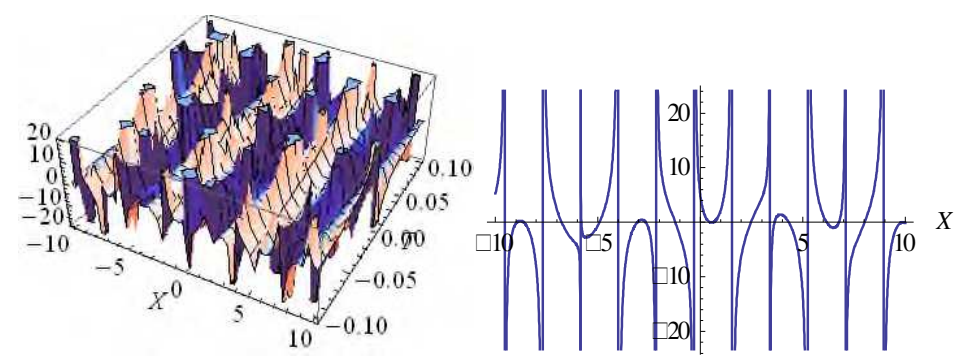

Fig.4 The cubic-quartic soliton of the Im. part of kerr-low Eq.(48) in 2D and 3D with values: $w_{Y}=-8$ $A_{-1}=A_{0}=0, A_{1}=1,\left|a_{0}\right|=9.6,\left|a_{2}\right|=0.3, k_{1}=b_{1}=d_{1}=q_{1}=\zeta_{0}=C_{0}=1, c_{1}=-25, \delta_{1}=-4.8-0.6 i, t=0.1$ for $2 \mathrm{D}$

Via the same manner, we can plot the other two cases.

Case 2: The second family, in which $a_{0}=a_{3}=0 \Rightarrow \varphi^{\prime}=a_{1} \varphi+a_{2} \varphi^{2}$, consequently

$$
\begin{aligned}
& \psi_{1}^{\prime}=A_{1} a_{2} \varphi^{2}+a_{1} A_{1} \varphi-\frac{A_{-1} a_{1}}{\varphi}-A_{-1} a_{2}, \\
& \psi_{1}^{\prime \prime \prime}=6 A_{1} a_{2}^{3} \varphi^{4}+12 a_{1} A_{1} a_{2}^{2} \varphi^{3}+7 A_{1} a_{1}^{2} a_{2} \varphi^{2}+A_{1} a_{1}^{3} \varphi-A_{-1} a_{2} a_{1}^{2}-\frac{a_{1}^{3} A_{-1}}{\varphi},
\end{aligned}
$$

Via inserting $\psi_{1}^{\prime \prime \prime}, \psi_{1}^{\prime} \psi_{1}^{2}$ and $\psi_{1}^{4}$ into equation (31),collecting and equating the coefficients of various powers of $\varphi^{i}$ to zero implies a system of equations, by solving it many solutions will be achieved from which only 8-results are suitable while the remaining are refused,

$$
\begin{aligned}
& a_{1}=\frac{2 \sqrt{3} q_{1}}{k_{1}}, a_{2}=\frac{\sqrt{3} A_{1} q_{1}}{A_{0} k_{1}}, \delta_{1}=-3\left(\frac{12 \sqrt{3} b_{1} k_{1} q_{1}^{3}+A_{0} b_{1} q_{1}^{4}}{A_{0}}\right), c_{1}=-d_{1}-\frac{72 b_{1} q_{1}^{3} \sqrt{3}}{\sqrt{k_{1}} A_{0}^{3}}, A_{-1}=0 \\
& a_{1}=\frac{-2 \sqrt{3} q_{1}}{k_{1}}, a_{2}=\frac{-\sqrt{3} A_{1} q_{1}}{A_{0} k_{1}}, \delta_{1}=-3\left(\frac{-12 \sqrt{3} b_{1} k_{1} q_{1}^{3}+A_{0} b_{1} q_{1}^{4}}{A_{0}}\right), c_{1}=-d_{1}+\frac{72 b_{1} q_{1}^{3} \sqrt{3}}{\sqrt{k_{1}} A_{0}^{3}}, A_{-1}=0 \\
& a_{1}=\frac{i q_{1} \sqrt{6}}{k_{1}}, a_{2}=0, \delta_{1}=-3 b_{1} q_{1}^{4}, c_{1}=-d_{1} \\
& a_{1}=\frac{-i q_{1} \sqrt{6}}{k_{1}}, a_{2}=0, \delta_{1}=-3 b_{1} q_{1}^{4}, c_{1}=-d_{1} \\
& a_{1}=\frac{-i q_{1} \sqrt{6}}{k_{1}}, \delta_{1}=-3 b_{1} q_{1}^{4}, c_{1}=-d_{1}, A_{1}=0
\end{aligned}
$$




$$
\begin{aligned}
& a_{1}=\frac{i q_{1} \sqrt{6}}{k_{1}}, \delta_{1}=-3 b_{1} q_{1}^{4}, c_{1}=-d_{1}, A_{1}=0 \\
& a_{1}=\frac{i q_{1} \sqrt{6}}{k_{1}}, a_{2}=0, \delta_{1}=-3 b_{1} q_{1}^{4}, c_{1}=-d_{1}, A_{-1}=0 \\
& a_{1}=\frac{-i q_{1} \sqrt{6}}{k_{1}}, a_{2}=0, \delta_{1}=-3 b_{1} q_{1}^{4}, c_{1}=-d_{1}, A_{-1}=0
\end{aligned}
$$

From which we can get 8-various solutions, we will plot some cases say, the first, the fifth and the eight results.

For the 1-st result which is,

$$
a_{1}=\frac{2 \sqrt{3} q_{1}}{k_{1}}, a_{2}=\frac{\sqrt{3} A_{1} q_{1}}{A_{0} k_{1}}, \delta_{1}=-3\left(\frac{12 \sqrt{3} b_{1} k_{1} q_{1}^{3}+A_{0} b_{1} q_{1}^{4}}{A_{0}}\right), c_{1}=-d_{1}-\frac{72 b_{1} q_{1}^{3} \sqrt{3}}{\sqrt{k_{1}} A_{0}^{3}}, A_{-1}=0
$$

This result can be simplified to be,

$$
A_{-1}=0, A_{0}=3, A_{1}=1, a_{1}=3.5, a_{2}=0.6, k_{1}=q_{1}=d_{1}=\zeta_{0}=1, c_{1}=-5.6, \delta_{1}=-23.8
$$

From the point of view of the suggested method the solution is,

$$
\begin{aligned}
& \varphi(\zeta)=\frac{a_{1} \operatorname{Exp}\left[a_{1}\left(\zeta+\zeta_{0}\right)\right]}{1-a_{2} \operatorname{Exp}\left[a_{1}\left(\zeta+\zeta_{0}\right)\right]}, a_{1} \mathrm{f} 0 \\
& \varphi(\zeta)=\frac{3.5 \operatorname{Exp}[3.5(x-8 t+1)]}{1-0.6 \operatorname{Exp}[3.5(x-8 t+1)]} \\
& \psi_{1}(\zeta)=3+\frac{3.5 \operatorname{Exp}[3.5(x-8 t+1)]}{1-0.6 \operatorname{Exp}[3.5(x-8 t+1)]} \\
& u(x, t)=\psi_{1}(\zeta) e^{i \eta_{1}(x, t)}, \zeta=k_{1} x+w_{1} t, \eta_{1}=q_{1} x+\delta_{1} t \\
& u(x, t)=\left(3+\frac{3.5 \operatorname{Exp}[3.5(x-8 t+1)]}{1-0.6 \operatorname{Exp}[3.5(x-8 t+1)]}\right) e^{i(x-23.8 t)} \\
& \operatorname{Re} u=\left(3+\frac{3.5 \operatorname{Exp}[3.5(x-8 t+1)]}{1-0.6 \operatorname{Exp}[3.5(x-8 t+1)]}\right) \times \cos (x-23.8 t) \\
& \operatorname{Im} u=\left(3+\frac{3.5 \operatorname{Exp}[3.5(x-8 t+1)]}{1-0.6 \operatorname{Exp}[3.5(x-8 t+1)]}\right) \times \sin (x-23.8 t)
\end{aligned}
$$

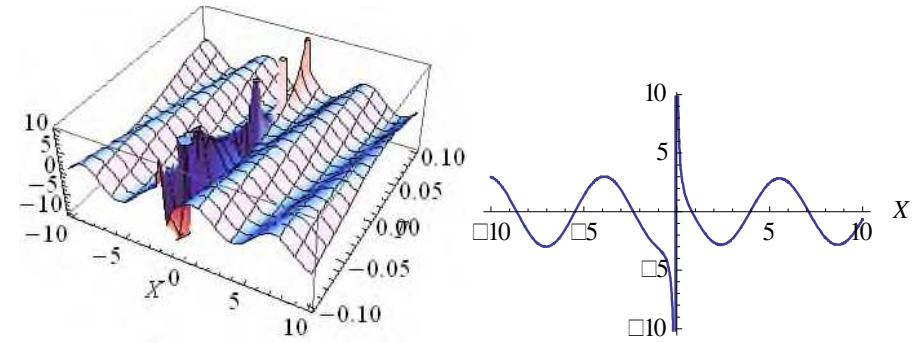

Fig.5 The cubic-quartic soliton of the Re. part of kerr-low Eq.(62) in 2D and 3D with values: $w_{1}=-8$

$$
A_{-1}=0, A_{0}=3, A_{1}=1, a_{1}=3.5, a_{2}=0.6, k_{1}=q_{1}=d_{1}=\zeta_{0}=1, c_{1}=-5.6, \delta_{1}=-23.8, t=0.1 \text { for } 2 \mathrm{D}
$$




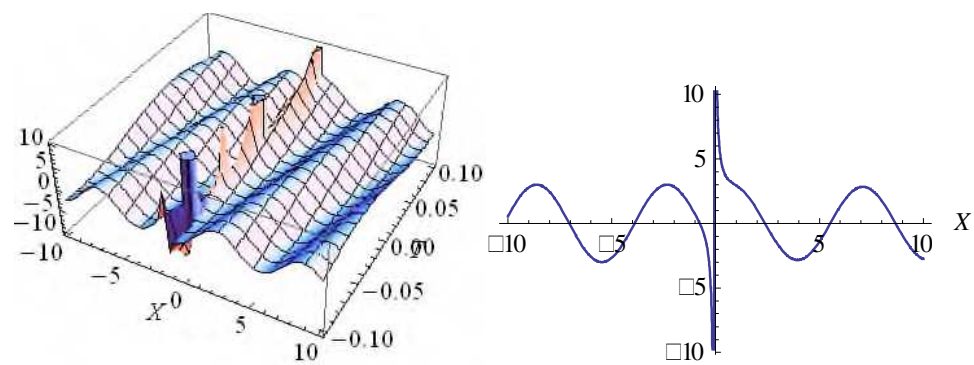

Fig.6 The cubic-quartic soliton of the Im. part of kerr-low Eq.(63) in 2D and 3D with values: $w=-8$

$$
A_{-1}=0, A_{0}=3, A_{1}=1, a_{1}=3.5, a_{2}=0.6, k_{1}=q_{1}=d_{1}=\zeta_{0}=1, c_{1}=-5.6, \delta_{1}=-23.8, t=0.1 \text { for } 2 \mathrm{D}
$$

For the fifth result which is, $a_{1}=\frac{-i q_{1} \sqrt{6}}{k_{1}}, \delta_{1}=-3 b_{1} q_{1}^{4}, c_{1}=-d_{1}, A_{1}=0$,

This result can be simplified to be,

$$
\begin{aligned}
A_{-1} & =1, A_{0}=1, A_{1}=0, a_{1}=-2.4 i, a_{2}=0, k_{1}=w_{1}=q_{1}=d_{1}=\zeta_{0}=c_{0}=1, c_{1}=-1, \delta_{1}=-3 \\
\varphi(\zeta) & =\frac{-a_{1} \operatorname{Exp}\left[a_{1}\left(\zeta+\zeta_{0}\right)\right]}{1+a_{2} \operatorname{Exp}\left[a_{1}\left(\zeta+\zeta_{0}\right)\right]}, a_{1} \mathrm{p} 0 \\
\varphi(\zeta) & =-2.4 i \operatorname{Exp}[-2.4 i(x-8 t+1)] \\
\psi_{1}(\zeta) & =\frac{1}{-2.4 i \operatorname{Exp}[-2.4 i(x-8 t+1)]}+1 \\
\psi_{1}(\zeta) & =0.4 i \operatorname{Exp}[2.4 i(x-8 t+1)]+1 \\
u(x, t) & =\psi_{1}(\zeta) e^{i_{1}(x, t)}, \zeta=k_{1} x+w_{1} t, \eta=q_{1} x+\delta_{1} t \\
u(x, t) & =([1-0.4 \sin (2.4 x-19.2 t+2.4)]+0.4 i \cos (2.4 x-19.2 t+2.4)) e^{i(x-3 t)} \\
\operatorname{Re} u & =((1-0.4 \sin [2.4 x-19.2 t+2.4]) \cos [x-3 t]-0.4 \cos [2.4 x-19.2 t+2.4] \sin [x-3 t]) \\
\operatorname{Im} u & =((1-0.4 \sin [2.4 x-19.2 t+2.4]) \sin [x-3 t]+0.4 \cos [2.4 x-19.2 t+2.4] \cos [x-3 t])
\end{aligned}
$$

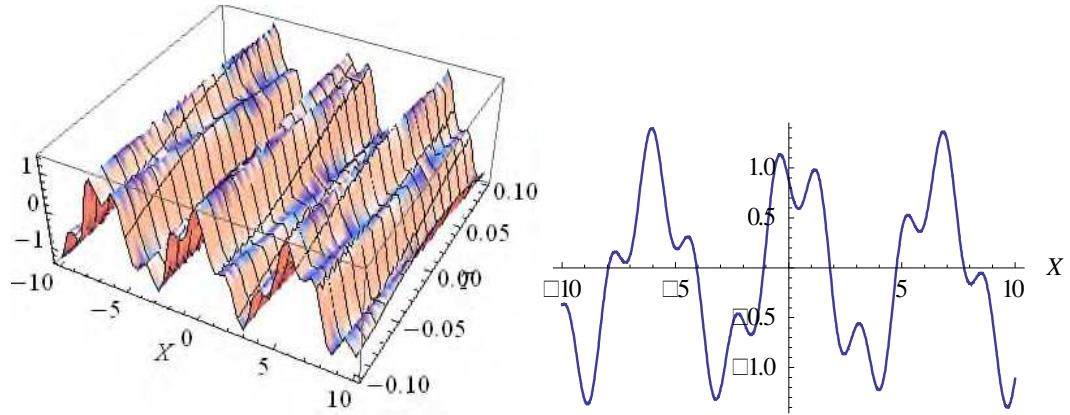

Fig.7 The cubic-quartic soliton of the Re. part of kerr-low Eq.(68) in 2D and 3D with values: $w=-8$

$$
A_{-1}=1, A_{0}=1, A_{1}=0, a_{1}=-2.4 i, a_{2}=0, k_{1}=q_{1}=d_{1}=\zeta_{0}=1, c_{1}=-1, \delta_{1}=-3, t=0.1 \text { for } 2 \mathrm{D}
$$




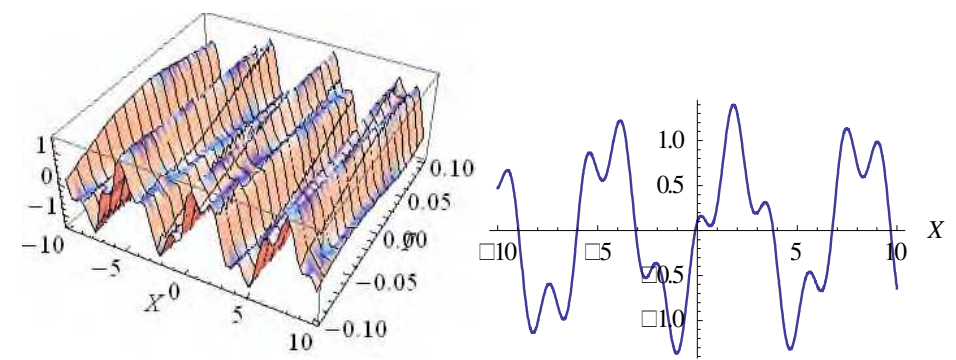

Fig.8 The cubic-quartic soliton of the Im. part of kerr-low Eq.(69) in 2D and 3D with values: $y_{1}=-8$

$$
A_{-1}=1, A_{0}=1, A_{1}=0, a_{1}=-2.4 i, a_{2}=0, k_{1}=q_{1}=d_{1}=\zeta_{0}=1, c_{1}=-1, \delta_{1}=-3, t=0.1 \text { for } 2 \mathrm{D}
$$

For the 8-th result which is, $a_{1}=\frac{-i q_{1} \sqrt{6}}{k_{1}}, a_{2}=0, \delta_{1}=-3 b_{1} q_{1}^{4}, c_{1}=-d_{1}, A_{-1}=0$,

This result can be simplified to be,

$$
\begin{aligned}
& A_{-1}=0, A_{0}=1, A_{1}=1, a_{1}=2.4 i, a_{2}=0, k_{1}=q_{1}=d_{1}=\zeta_{0}=1, c_{1}=-1, \delta_{1}=-3 \\
& \varphi(\zeta)= \frac{a_{1} \operatorname{Exp}\left[a_{1}\left(\zeta+\zeta_{0}\right)\right]}{1-a_{2} \operatorname{Exp}\left[a_{1}\left(\zeta+\zeta_{0}\right)\right]}, a_{1} \mathrm{f} 0 \\
& \varphi(\zeta)= 2.4 i \operatorname{Exp}[2.4 i(x-8 t+1)] \\
& \psi_{1}(\zeta)=[1-2.4 \sin (2.4 x-19.2 t+2.4)]+2.4 i \cos (2.4 x-19.2 t+2.4) \\
& u(x, t)=([1-2.4 \sin (2.4 x-19.2 t+2.4)]+2.4 i \cos (2.4 x-19.2 t+2.4)) e^{i(x-3 t)}
\end{aligned}
$$

$\operatorname{Im} u=(2.4 \cos (2.4 x-19.2 t+2.4) \times \cos (x-3 t)+2.4[(1-2.4 \sin (2.4 x-19.2 t+2.4)) \times \sin (x-3 t))$
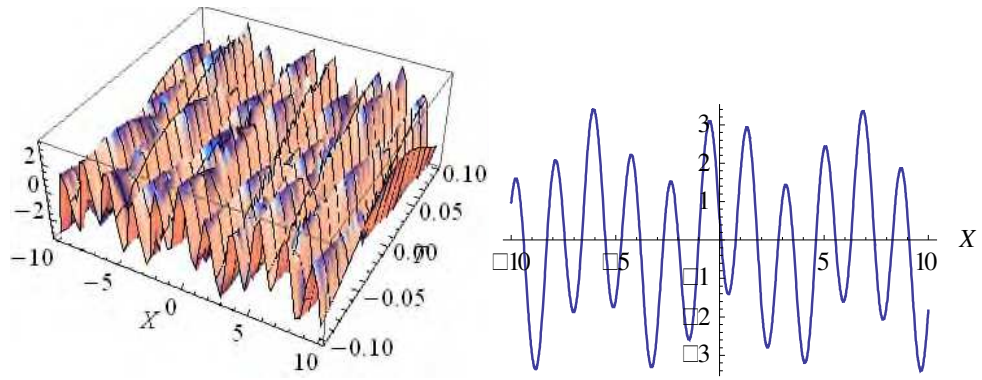

Fig.9 The cubic-quartic soliton of the Re. part of kerr-low Eq.(73) in 2D and 3D with values: $w=-8$

$$
A_{-1}=0, A_{0}=1, A_{1}=1, a_{1}=2.4 i, a_{2}=0, k_{1}=q_{1}=d_{1}=\zeta_{0}=1, c_{1}=-1, \delta_{1}=-3, t=0.1 \text { for } 2 \mathrm{D}
$$
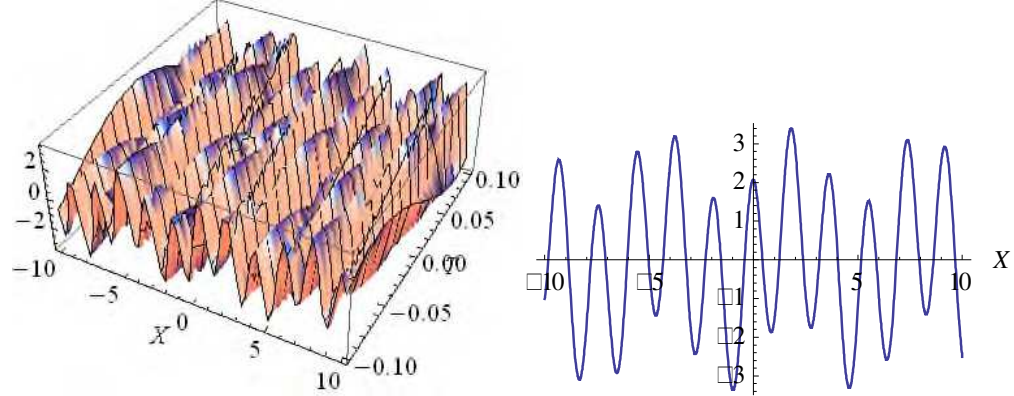

Fig.10 The cubic-quartic soliton of the Im. part of kerr-low Eq.(74) in 2D and 3D with values: $w_{1}=-8$ values: $A_{-1}=0, A_{0}=1, A_{1}=1, a_{1}=2.4 i, a_{2}=0, k_{1}=q_{1}=d_{1}=\zeta_{0}=1, c_{1}=-1, \delta_{1}=-3, t=0.1$ for $2 \mathrm{D}$ In the same manner, we can plot the other cases. 


\section{The Cubic-Quartic NLSE for the Quadratic-Low nonlinearity using the ESEM}

The cubic-quartic NLSE in polarization-preserving fibers with quadratic-cubic law can be proposed in the form,

$$
i Q_{t}+i a Q_{x x x}+b Q_{x x x x}=\left(c|Q|+d\left|Q^{2}\right|\right) Q
$$

The cubic-quartic NLSE in birefringent fiber for quadratic-cubic law is,

$$
\begin{aligned}
& i u_{t}+i a_{1} u_{x x x}+b_{1} u_{x x x x}+c_{1} u \sqrt{|u|^{2}+|v|^{2}+u v^{*}+v u^{*}}+\left(d_{1}|u|^{2}+e_{1}|v|^{2}\right) u=0 \\
& i v_{t}+i a_{2} v_{x x x}+b_{2} v_{x x x x}+c_{2} v \sqrt{|v|^{2}+|u|^{2}+u v^{*}+v u^{*}}+\left(d_{2}|v|^{2}+e_{2}|u|^{2}\right) v=0
\end{aligned}
$$

Via inserting the relations (13-26) into equation (76) and (77) will transform them to the following real and imaginary equations respectively:

$$
\begin{gathered}
\operatorname{Re} \Rightarrow b_{j} k_{j}^{4} \psi_{j}^{\prime \prime \prime \prime}-\left(3 a_{j} q_{j} k_{j}^{2}+6 k_{j}^{2} q_{j}^{2}\right) \psi_{j}^{\prime \prime}+\left(b_{j} q_{j}^{4}+a_{j} q_{j}^{3}-\delta_{j}\right) \psi_{j}+2 c_{j} \psi_{j}^{2}+\left(d_{j}+e_{j}\right) \psi_{j}^{3}=0 \\
\operatorname{Im} \Rightarrow k_{j}^{3}\left(a_{j}+4 b_{j} q_{j}\right) \psi_{j}^{\prime \prime \prime}+\left(w_{j}-3 a_{j} k_{j} q_{j}^{2}-4 k_{j} q_{j}^{3}\right) \psi_{j}^{\prime}=0
\end{gathered}
$$

Eq. (79) implies $a_{j}=-4 b_{j} q_{j}, w_{j}=3 a_{j} q_{j}^{2}+4 b_{j} q_{j}^{3}$, hence under this constrain Eq.(78) will be converted to,

$$
b_{j} k_{j}^{4} \psi_{j}^{\prime \prime \prime \prime}+6 b_{j} k_{j}^{2} q_{j}^{2} \psi_{j}^{\prime \prime}-\left(3 b_{j} q_{j}^{4}+\delta_{j}\right) \psi_{j}+2 c_{j} \psi_{j}^{2}+\left(d_{j}+e_{j}\right) \psi_{j}^{3}=0
$$

Equation (80) represents either the first part Eq.(76) or the second part Eq.(77) which is the same, for similarity we will implement the solution for the first part $j=1$ which is,

$$
b_{1} k_{1}^{4} \psi_{1}^{\prime \prime \prime}+6 b_{1} k_{1}^{2} q_{1}^{2} \psi_{1}^{\prime \prime}-\left(3 b_{1} q_{1}^{4}+\delta_{1}\right) \psi_{1}+2 c_{1} \psi_{1}^{2}+\left(d_{1}+e_{1}\right) \psi_{1}^{3}=0
$$

Now, via integrating Eq. (81) with respect to $\zeta$ it becomes,

$$
b_{1} k_{1}^{4} \psi_{1}^{\prime \prime \prime}+6 b_{1} k_{1}^{2} q_{1}^{2} \psi_{1}^{\prime}-\frac{1}{2}\left(3 b_{1} q_{1}^{4}+\delta_{1}\right) \psi_{1}^{2}+\frac{2}{3} c_{1} \psi_{1}^{3}+\frac{1}{4}\left(d_{1}+e_{1}\right) \psi_{1}^{4}=0
$$

Via balancing $\psi_{1}^{\prime \prime \prime}, \psi_{1}^{4}$ at Eq.(82) leads to $4 N=N+3$ which implies that $N=1$, hence the solution is:

$$
\psi_{1}(\zeta)=\frac{A_{-1}}{\varphi}+A_{0}+A_{1} \varphi
$$

Where $\varphi^{\prime}=a_{0}+a_{1} \varphi+a_{2} \varphi^{2}+a_{3} \varphi^{3}$

Case 1: The first family, in which $a_{1}=a_{3}=0 \Rightarrow \varphi^{\prime}=a_{0}+a_{2} \varphi^{2}$, consequently

$$
\begin{aligned}
\psi_{1}^{\prime} & =-\frac{a_{0} A_{-1}}{\varphi^{2}}+A_{1} a_{0}+A_{1} a_{2} \varphi^{2}-a_{2} A_{-1} \\
\psi_{1}^{\prime \prime \prime} & =\frac{-6 a_{0}^{3} A_{-1}}{\varphi^{4}}-\frac{8 a_{0}^{2} a_{2} A_{-1}}{\varphi^{2}}-2 A_{-1} a_{0} a_{2}^{2}+2 A_{1} a_{2} a_{0}^{2}+8 A_{1} a_{0} a_{2}^{2} \varphi^{2}+6 A_{1} a_{2}^{3} \varphi^{4}
\end{aligned}
$$

Via inserting $\psi_{1}^{\prime \prime \prime}, \psi_{1}^{\prime}, \psi_{1}^{2}$ and $\psi_{1}^{4}$ into equation (82), collecting and equating the coefficients of various powers of $\varphi^{i}$ to zero leads to system of equations from which we construct that there are no solutions achieved because either $a_{0}=0$ or $a_{2}=0$ or both or $A_{-1}=A_{1}=0$.

Case 2: The second family, in which $a_{0}=a_{3}=0 \Rightarrow \varphi^{\prime}=a_{1} \varphi+a_{2} \varphi^{2}$, consequently 


$$
\psi_{1}^{\prime}=A_{1} a_{2} \varphi^{2}+a_{1} A_{1} \varphi-\frac{A_{-1} a_{1}}{\varphi}-A_{-1} a_{2}
$$

$\psi_{1}^{\prime \prime \prime}=6 A_{1} a_{2}^{3} \varphi^{4}+12 a_{1} A_{1} a_{2}^{2} \varphi^{3}+7 A_{1} a_{1}^{2} a_{2} \varphi^{2}+A_{1} a_{1}^{3} \varphi-A_{-1} a_{2} a_{1}^{2}-\frac{a_{1}^{3} A_{-1}}{\varphi}$

Via inserting $\psi_{1}^{\prime \prime \prime}, \psi_{1}^{\prime}, \psi_{1}^{2}, \psi_{1}^{3}$ and $\psi_{1}^{4}$ into equation (82), collecting and equating the coefficients of various powers of $\varphi^{i}$ to zero leads to system of equations which proposed big number of very complicated solutions from which only 12 -solutions are valued and the other achieved solutions are refused because either $a_{1}=0$ or $A_{-1}=A_{1}=0$, we will choose only 8solutions of them where the remaining are very long which are,

$$
\begin{aligned}
& A_{-1}=0, a_{1}=\frac{i \sqrt{6} q_{1}}{k_{1}}, a_{2}=\frac{i \sqrt{6} A_{1} q_{1}}{A_{0} k_{1}}, b_{1}=\frac{-i A_{0}^{3}\left(d_{1}+e_{1}\right)}{144 \sqrt{6} k_{1} q_{1}^{3}}, c_{1}=-\frac{3}{4} A_{0}\left(d_{1}+e_{1}\right), \\
& \delta_{1}=\frac{i\left[144 i A_{0}^{2} k_{1}\left(d_{1}+e_{1}\right)+\sqrt{6} A_{0}^{3} q_{1}\left(d_{1}+e_{1}\right)\right]}{288 k_{1}} . \\
& A_{-1}=0, a_{1}=\frac{-i \sqrt{6} q_{1}}{k_{1}}, a_{2}=\frac{-i \sqrt{6} A_{1} q_{1}}{A_{0} k_{1}}, b_{1}=\frac{-i A_{0}^{3}\left(d_{1}+e_{1}\right)}{144 \sqrt{6} k_{1} q_{1}^{3}}, c_{1}=-\frac{3}{4} A_{0}\left(d_{1}+e_{1}\right), \\
& \delta_{1}=\frac{i\left[-144 i A_{0}^{2} k_{1}\left(d_{1}+e_{1}\right)+\sqrt{6} A_{0}^{3} q_{1}\left(d_{1}+e_{1}\right)\right]}{288 k_{1}} . \\
& A_{-1}=0, A_{0}=0, a_{1}=\frac{i \sqrt{6} q_{1}}{k_{1}}, a_{2}=\frac{(-1)^{\frac{1}{3}} A_{1}\left(d_{1}+e_{1}\right)^{\frac{1}{3}}}{2\left(b_{1}\right)^{\frac{1}{3}}\left(k_{1}\right)^{\frac{4}{3}}}, c_{1}=-\frac{(-1)^{\frac{2}{3}}\left(3 b_{1} k_{1}\right)^{\frac{1}{3}}\left[3 i \sqrt{6} q_{1}\left(d_{1}+e_{1}\right)\right]}{2\left(d_{1}+e_{1}\right)^{\frac{1}{3}}}, \\
& \delta_{1}=\frac{3\left[-4(-1)^{\frac{1}{3}}\left(3 b_{1} k_{1}\right)^{\frac{2}{3}} d_{1}^{2} q_{1}^{2}-8(-1)^{\frac{1}{3}}\left(3 b_{1} k_{1}\right)^{\frac{1}{3}} d_{1} e_{1} q_{1}^{2}-4(-1)^{\frac{1}{3}}\left(3 b_{1} k_{1}\right)^{\frac{2}{3}} e_{1}^{2} q_{1}^{2}\right]}{5}-b_{1} q_{1}^{4} \\
& \left(d_{1}+e_{1}\right)^{\frac{5}{3}} \\
& A_{-1}=0, A_{6}=0, a_{1}=\frac{-i \sqrt{6} q_{1}}{k_{1}}, a_{2}=\frac{(-1)^{\frac{1}{3}} A_{1}\left(d_{1}+e_{1}\right)^{\frac{1}{3}}}{2\left(b_{1}\right)^{\frac{1}{3}}\left(k_{1}\right)^{\frac{4}{3}}}, c_{1}=-\frac{(-1)^{\frac{2}{3}}\left(3 b_{1} k_{1}\right)^{\frac{1}{3}}\left[3 i \sqrt{6} q_{1}\left(d_{1}+e_{1}\right)\right]}{2\left(d_{1}+e_{1}\right)^{\frac{1}{3}}}, \\
& \delta_{1}=\frac{3\left[-4(-1)^{\frac{1}{3}}\left(3 b_{1} k_{1}\right)^{\frac{2}{3}} d_{1}^{2} q_{1}^{2}-8(-1)^{\frac{1}{3}}\left(3 b_{1} k_{1}\right)^{\frac{1}{3}} d_{1} e_{1} q_{1}^{2}-4(-1)^{\frac{1}{3}}\left(3 b_{1} k_{1}\right)^{\frac{2}{3}} e_{1}^{2} q_{1}^{2}\right]}{y^{\frac{5}{3}}}-b_{1} q_{1}^{4} \\
& \left(d_{1}+e_{1}\right)^{\overline{3}} \\
& A_{-1}=0, A_{0}=0, a_{1}=\frac{i \sqrt{6} q_{1}}{k_{1}}, a_{2}=\frac{-A_{1}\left(d_{1}+e_{1}\right)^{\frac{1}{3}}}{2\left(b_{1}\right)^{\frac{1}{3}}\left(k_{1}\right)^{\frac{4}{3}}}, c_{1}=-\frac{\left(3 b_{1} k_{1}\right)^{\frac{1}{3}}\left[3 i \sqrt{6} q_{1}\left(d_{1}+e_{1}\right)\right]}{2\left(d_{1}+e_{1}\right)^{\frac{1}{3}}}, \\
& \delta_{1}=\frac{3\left[4\left(3 b_{1} k_{1}\right)^{\frac{2}{3}} d_{1}^{2} q_{1}^{2}+8\left(3 b_{1} k_{1}\right)^{\frac{1}{3}} d_{1} e_{1} q_{1}^{2}+4\left(3 b_{1} k_{1}\right)^{\frac{2}{3}} e_{1}^{2} q_{1}^{2}\right]}{\frac{5}{3}}-b_{1} q_{1}^{4} \\
& \left(d_{1}+e_{1}\right)^{\overline{3}}
\end{aligned}
$$




$$
\begin{aligned}
& A_{-1}=0, A_{0}=0, a_{1}=\frac{-i \sqrt{6} q_{1}}{k_{1}}, a_{2}=\frac{-A_{1}\left(d_{1}+e_{1}\right)^{\frac{1}{3}}}{2\left(b_{1}\right)^{\frac{1}{3}}\left(k_{1}\right)^{\frac{4}{3}}}, c_{1}=\frac{\left(3 b_{1} k_{1}\right)^{\frac{1}{3}}\left[3 i \sqrt{6} q_{1}\left(d_{1}+e_{1}\right)\right]}{2\left(d_{1}+e_{1}\right)^{\frac{1}{3}}}, \\
& \delta_{1}=\frac{3\left[4\left(3 b_{1} k_{1}\right)^{\frac{2}{3}} d_{1}^{2} q_{1}^{2}+8\left(3 b_{1} k_{1}\right)^{\frac{1}{3}} d_{1} e_{1} q_{1}^{2}+4\left(3 b_{1} k_{1}\right)^{\frac{2}{3}} e_{1}^{2} q_{1}^{2}\right]}{\left(d_{1}+e_{1}\right)^{\frac{5}{3}}}-b_{1} q_{1}^{4} \\
& A_{-1}=0, A_{0}=0, a_{1}=\frac{i \sqrt{6} q_{1}}{k_{1}}, a_{2}=\frac{-A_{1}\left(d_{1}+e_{1}\right)^{\frac{1}{3}}}{2\left(b_{1}\right)^{\frac{1}{3}}\left(k_{1}\right)^{\frac{4}{3}}}, c_{1}=\frac{\left(-3 b_{1} k_{1}\right)^{\frac{1}{3}}\left[3 i \sqrt{6} q_{1}\left(d_{1}+e_{1}\right)\right]}{2\left(d_{1}+e_{1}\right)^{\frac{1}{3}}}, \\
& \delta_{1}=\frac{3\left[4\left(-3 b_{1} k_{1}\right)^{\frac{2}{3}} d_{1}^{2} q_{1}^{2}+8\left(-3 b_{1} k_{1}\right)^{\frac{1}{3}} d_{1} e_{1} q_{1}^{2}+4\left(-3 b_{1} k_{1}\right)^{\frac{2}{3}} e_{1}^{2} q_{1}^{2}\right]}{\left(d_{1}+e_{1}\right)^{\frac{5}{3}}}-b_{1} q_{1}^{4} \\
& A_{-1}=0, A_{0}=0, a_{1}=\frac{-i \sqrt{6} q_{1}}{k_{1}}, a_{2}=\frac{-A_{1}\left(d_{1}+e_{1}\right)^{\frac{1}{3}}}{2\left(b_{1}\right)^{\frac{1}{3}}\left(k_{1}\right)^{\frac{4}{3}}}, c_{1}=-\frac{\left(-3 b_{1} k_{1}\right)^{\frac{1}{3}}\left[3 i \sqrt{6} q_{1}\left(d_{1}+e_{1}\right)\right]}{2\left(d_{1}+e_{1}\right)^{\frac{1}{3}}}, \\
& \delta_{1}=\frac{3\left[4\left(-3 b_{1} k_{1}\right)^{\frac{2}{3}} d_{1}^{2} q_{1}^{2}+8\left(-3 b_{1} k_{1}\right)^{\frac{1}{3}} d_{1} e_{1} q_{1}^{2}+4\left(-3 b_{1} k_{1}\right)^{\frac{2}{3}} e_{1}^{2} q_{1}^{2}\right]}{\left(d_{1}+e_{1}\right)^{\frac{5}{3}}}-b_{1} q_{1}^{4}
\end{aligned}
$$

For simplicity we will choose only one of these results say the first one, to extract its own solution and plotted it.

For the 1-th result which is,

$$
\begin{aligned}
& A_{-1}=0, a_{1}=\frac{i \sqrt{6} q_{1}}{k_{1}}, a_{2}=\frac{i \sqrt{6} A_{1} q_{1}}{A_{0} k_{1}}, b_{1}=\frac{-i A_{0}^{3}\left(d_{1}+e_{1}\right)}{144 \sqrt{6} k_{1} q_{1}^{3}}, c_{1}=-\frac{3}{4} A_{0}\left(d_{1}+e_{1}\right), \\
& \delta_{1}=\frac{-A_{0}^{2}\left(d_{1}+e_{1}\right)}{2}+i \frac{\sqrt{6} A_{0}^{3} q_{1}\left(d_{1}+e_{1}\right)}{288 k_{1}} .
\end{aligned}
$$

This result will simplified to be,

$$
A_{-1}=0, A_{0}=4, A_{1}=1, a_{1}=0.6 i, a_{2}=0.6 i, b_{1}=-0.4 i, e_{1}=d_{1}=k_{1}=q_{1}=\zeta_{0}=1, c_{1}=-6, \delta_{1}=-16+1.1 i
$$

Via these values of constants the solution in the framework of the proposed method is,

$$
\begin{gathered}
\varphi(\zeta)=\frac{a_{1} \operatorname{Exp}\left[a_{1}\left(\zeta+\zeta_{0}\right)\right]}{1-a_{2} \operatorname{Exp}\left[a_{1}\left(\zeta+\zeta_{0}\right)\right]}, a_{1} \mathrm{f} 0 \\
\varphi(\zeta)=\frac{i \sqrt{6} \operatorname{Exp}[i \sqrt{6}(x+t+1)]}{1-i \sqrt{6} \operatorname{Exp}[i \sqrt{6}(x+t+1)]} \\
\psi_{1}(\zeta)=4+\frac{i \sqrt{6} \operatorname{Exp}[i \sqrt{6}(x+t+1)]}{1-i \sqrt{6} \operatorname{Exp}[i \sqrt{6}(x+t+1)]} \\
\psi_{1}(\zeta)=\frac{4-3 i \sqrt{6} \operatorname{Exp}[i \sqrt{6}(x+t+1)]}{1-i \sqrt{6} \operatorname{Exp}[i \sqrt{6}(x+t+1)]}
\end{gathered}
$$




$$
\begin{aligned}
& u(x, t)=\left(\frac{4-3 i \sqrt{6} \operatorname{Exp}[i \sqrt{6}(x+t+1)]}{1-i \sqrt{6} \operatorname{Exp}[i \sqrt{6}(x+t+1)]}\right) \times e^{i(x+1.1 i t-16 t)} \\
& u(x, t)=e^{-1.1 t}\left(\frac{4-3 i \sqrt{6} \operatorname{Exp}[i \sqrt{6}(x+t+1)]}{1-i \sqrt{6} \operatorname{Exp}[i \sqrt{6}(x+t+1)]}\right) \times e^{i(x-16 t)} \\
& \operatorname{Re} u=e^{-1.1 t}\left\{\begin{array}{l}
\frac{(22+7 \sqrt{6} \sin [\sqrt{6}(x+t+1)]) \cos (x-16 t)}{7+2 \sqrt{6} \sin [\sqrt{6}(x+t+1)]} \\
-\frac{\sqrt{6} \cos [\sqrt{6}(x+t+1)] \times \sin (x-16)}{7+2 \sqrt{6} \sin [\sqrt{6}(x+t+1)]}
\end{array}\right\} \\
& \operatorname{Im} u=e^{-1.1 t}\left\{\begin{array}{l}
\frac{(22+7 \sqrt{6} \sin [\sqrt{6}(x+t+1)]) \sin (x-16 t)}{7+2 \sqrt{6} \sin [\sqrt{6}(x+t+1)]} \\
+\frac{\sqrt{6} \cos [\sqrt{6}(x+t+1)] \times \cos (x-16)}{7+2 \sqrt{6} \sin [\sqrt{6}(x+t+1)]}
\end{array}\right\}
\end{aligned}
$$

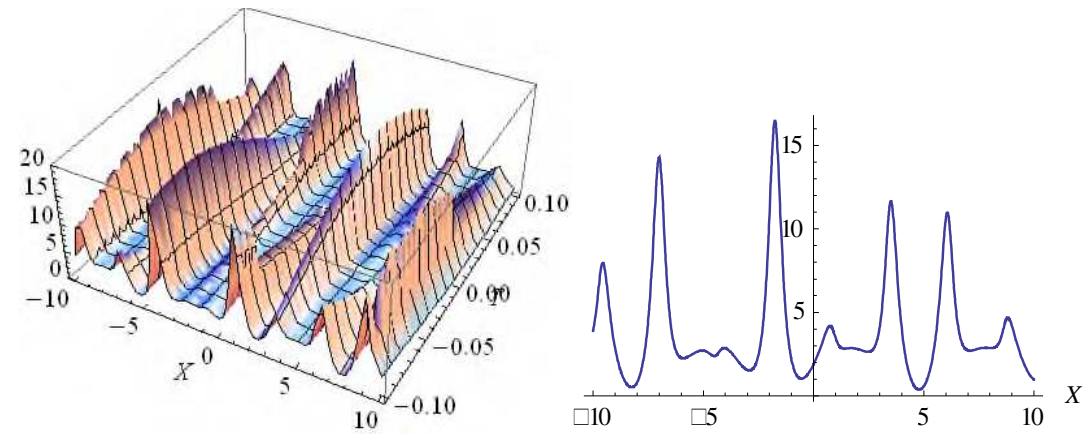

Fig.11 The cubic-quartic soliton of the Re. part of quadratic-low Eq.(98) in 2D and 3D at: $w_{\mathrm{Y}}=-8$, $A_{-1}=0, A_{0}=4, A_{1}=1, a_{1}=0.6 i, a_{2}=0.6 i, b_{1}=-0.4 i, e_{1}=d_{1}=k_{1}=q_{1}=\zeta_{0}=1, c_{1}=-6, \delta_{1}=-16+1.1 i, t=0.1$ for $2 \mathrm{D}$
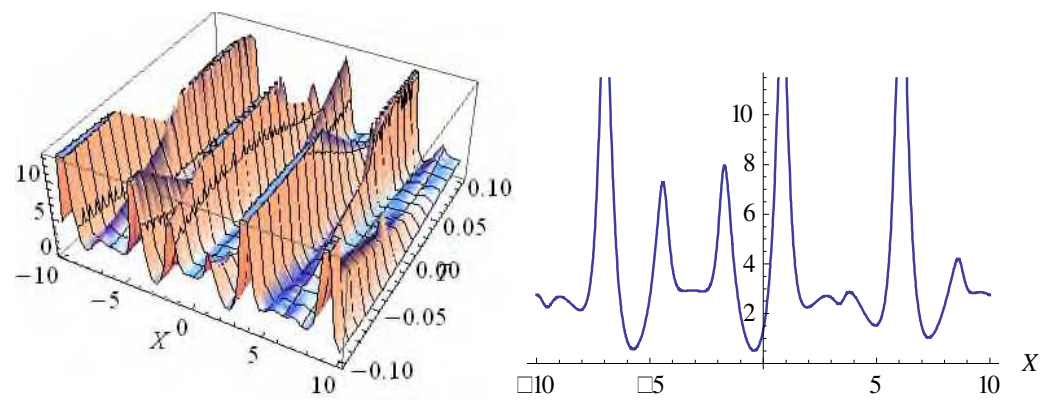

Fig.12 The cubic-quartic soliton of the Im. part of quadratic-low Eq.(99) in 2D and 3D at: $w_{1}=-8$, $A_{-1}=0, A_{0}=4, A_{1}=1, a_{1}=0.6 i, a_{2}=0.6 i, b_{1}=-0.4 i, e_{1}=d_{1}=k_{1}=q_{1}=\zeta_{0}=1, c_{1}=-6, \delta_{1}=-16+1.1 i, t=0.1$ for $2 \mathrm{D}$

5. The Cubic-Quartic NLSE for the Parabolic-Low nonlinearity using the ESEM The cubic-quartic NLSE in polarization-preserving fibers with parabolic law can be proposed in the form,

$$
i Q_{t}+i a Q_{x x x}+b Q_{x x x x}=\left(c\left|Q^{2}\right|+d\left|Q^{4}\right|\right) Q
$$


The cubic-quartic NLSE in birefringent fiber for parabolic-law is,

$$
\begin{aligned}
& i u_{t}+i a_{1} u_{x x x}+b_{1} u_{x x x x}+c_{1}|u|^{2} u+d_{1}|v|^{2} u+\left(e_{1}\left|u^{4}\right|+g_{1}\left|u^{2}\right|\left|v^{2}\right|+h_{1}\left|v^{4}\right|\right) u=0 \\
& i v_{t}+i a_{1} v_{x x x}+b_{1} v_{x x x x}+c_{1}|v|^{2} v+d_{1}|u|^{2} v+\left(e_{1}\left|v^{4}\right|+g_{1}\left|v^{2}\right|\left|u^{2}\right|+h_{1}\left|u^{4}\right|\right) v=0
\end{aligned}
$$

Via inserting the relations (13-26) into the equations (101) and (102) will transform them to the following real and imaginary equations respectively

$$
\begin{aligned}
& \operatorname{Re} \Rightarrow b_{j} k_{j}^{4} \psi_{j}^{\prime \prime \prime}-\left(3 a_{j} q_{j} k_{j}^{2}+6 b_{j} k_{j}^{2} q_{j}^{2}\right) \psi_{j}^{\prime \prime}+\left(b_{j} q_{j}^{4}+a_{j} q_{j}^{3}-\delta_{j}\right) \psi_{j}+\left(c_{j}+d_{j}\right) \psi_{j}^{3}+(e+g+h) \psi_{j}^{5}=0 \\
& \operatorname{Im} \Rightarrow k_{j}^{3}\left(a_{j}+4 b_{j} q_{j}\right) \psi_{j}^{\prime \prime \prime}+\left(w_{j}-3 a_{j} k_{j} q_{j}^{2}-4 b_{j} k_{j} q_{j}^{3}\right) \psi_{j}^{\prime}=0
\end{aligned}
$$

Equation (104) implies $a_{j}=-4 b_{j} q_{j}, w_{j}=3 k_{j} a_{j} q_{j}^{2}+4 k_{j} b_{j} q_{j}^{3}$, hence under this constrain Eq. (103) will be converted to,

$$
b_{j} k_{j}^{4} \psi_{j}^{\prime \prime \prime \prime}+6 b_{j} q_{j}^{2} k_{j}^{2} \psi_{j}^{\prime \prime}-\left(3 k_{j} b_{j} q_{j}^{4}+\delta_{j}\right) \psi_{j}+\left(c_{j}+d_{j}\right) \psi_{j}^{3}+(e+g+h) \psi_{j}^{5}=0
$$

Equation (105) represents either the first part Eq. (101) or the second part Eq. (102) which is the same, for similarity we will implement the solution for the first part $j=1$ which is,

$$
b_{1} k_{1}^{4} \psi_{1}^{\prime \prime \prime \prime}+6 b_{1} q_{1}^{2} k_{1}^{2} \psi_{1}^{\prime \prime}-\left(3 b_{1} q_{1}^{4}+\delta_{1}\right) \psi_{1}+\left(c_{1}+d_{1}\right) \psi_{1}^{3}+(e+g+h) \psi_{1}^{5}=0
$$

Via balancing $\psi_{1}^{\prime \prime \prime}, ' \psi_{1}^{5}$ at Eq. (106) leads to $5 N=N+4$ which implies that $N=1$, hence the solution is:

$$
\psi_{1}(\zeta)=\frac{A_{-1}}{\varphi}+A_{0}+A_{1} \varphi
$$

Where $\varphi^{\prime}=a_{0}+a_{1} \varphi+a_{2} \varphi^{2}+a_{3} \varphi^{3}$

Case 1: The first family, in which $a_{1}=a_{3}=0 \Rightarrow \varphi^{\prime}=a_{0}+a_{2} \varphi^{2}$, consequently

$$
\begin{gathered}
\psi_{1}^{\prime}=-\frac{a_{0} A_{-1}}{\varphi^{2}}+A_{1} a_{0}+A_{1} a_{2} \varphi^{2}-a_{2} A_{-1} \\
\psi_{1}^{\prime \prime}=\frac{2 a_{0}^{2} A_{-1}}{\varphi^{3}}+\frac{2 a_{0} a_{2} A_{-1}}{\varphi}+2 A_{1} a_{0} a_{2} \varphi+2 A_{1} a_{2}^{2} \varphi^{3} \\
\psi_{1}^{\prime \prime \prime \prime}=\frac{24 a_{0}^{4} A_{-1}}{\varphi^{5}}+\frac{40 a_{0}^{3} a_{2} A_{-1}}{\varphi^{3}}+\frac{16 a_{0}^{2} a_{2}^{2} A_{-1}}{\varphi}+16 A_{1} a_{0}^{2} a_{2}^{2} \varphi+40 A_{1} a_{0} a_{2}^{3} \varphi^{3}+24 A_{1} a_{2}^{4} \varphi^{5}
\end{gathered}
$$

Via inserting $\psi_{1}^{\prime \prime \prime}, \psi_{1}^{\prime \prime}, \psi_{1}, \psi_{1}^{3}$ and $\psi_{1}^{5}$ into equation (106), collecting and equating the coefficients of various powers of $\varphi^{i}$ to zero leads to system of equations, by solving this system many solutions will be achieved from which only 8-results are acceptable while the remaining are refused. These acceptable results are,

$$
\begin{aligned}
& A_{0}=0, a_{2}=\frac{a_{0} A_{1}}{A_{-1}}, \delta_{1}=\frac{b_{1} k_{1}\left(16 a_{0}^{4} A_{1}^{2} k_{1}^{3}-24 a_{0}^{2} A_{1} A_{-1} q_{1}^{2}-3 A_{-1}^{2} q_{1}^{2}\right)}{A_{-1}^{2}}, \\
& c_{1}=\frac{-A_{-1}^{3} d_{1}+80 a_{0}^{4} A_{1} b_{1} k_{1}^{4}-12 a_{0}^{2} A_{-1} b_{1} q_{1}^{2} k_{1}^{2}}{A_{-1}^{3}}, g=\frac{-(e+h) A_{-1}^{4}-24 b_{1} a_{0}^{4} k_{1}^{4}}{A_{-1}^{4}} \\
& A_{0}=0, a_{2}=\frac{-a_{0} A_{1}}{A_{-1}}, \delta_{1}=\frac{-b_{1}\left(-256 a_{0}^{4} A_{1}^{2} k_{1}^{4}+48 a_{0}^{2} A_{1} A_{-1} k_{1}^{2} q_{1}^{2}+3 A_{-1}^{2} q_{1}^{4}\right)}{A_{-1}^{2}}, \\
& c_{1}=\frac{-A_{-1}^{3} d_{1}+160 a_{0}^{4} A_{1} b_{1} k_{1}^{4}-12 a_{0}^{2} A_{-1} b_{1} k_{1}^{2} q_{1}^{2}}{A_{-1}^{3}}, g=\frac{-(e+h) A_{-1}^{4}-24 b_{1} a_{0}^{4} k_{1}^{4}}{A_{-1}^{4}}
\end{aligned}
$$




$$
\begin{aligned}
& A_{0}=0, a_{2}=\frac{i a_{0} A_{1}}{A_{-1}}, \delta_{1}=\frac{532 b_{1} a_{0}^{4} A_{1}^{2} k_{1}^{4}}{3 A_{-1}^{2}}, q_{1}=\frac{-(-1)^{\frac{3}{4}} k_{1} a_{0} \sqrt{\frac{10 A_{1}}{3}}}{\sqrt{A_{-1}}} \\
& c_{1}=\frac{-A_{-1}^{3} d_{1}+120 a_{0}^{4} A_{1} b_{1} k_{1}^{4}}{A_{-1}^{3}}, g=\frac{-(e+h) A_{-1}^{4}-24 b_{1} a_{0}^{4} k_{1}^{4}}{A_{-1}^{4}} \\
& A_{0}=0, a_{2}=\frac{i a_{0} A_{1}}{A_{-1}}, \delta_{1}=\frac{532 b_{1} a_{0}^{4} A_{1}^{2} k_{1}^{4}}{3 A_{-1}^{2}}, q_{1}=\frac{(-1)^{\frac{3}{4}} k_{1} a_{0} \sqrt{\frac{10 A_{1}}{3}}}{\sqrt{A_{-1}}} \\
& c_{1}=\frac{-A_{-1}^{3} d_{1}+120 a_{0}^{4} A_{1} b_{1} k_{1}^{4}}{A_{-1}^{3}}, g=\frac{-(e+h) A_{-1}^{4}-24 b_{1} a_{0}^{4} k_{1}^{4}}{A_{-1}^{4}} \\
& A_{0}=0, a_{2}=\frac{-i a_{0} A_{1}}{A_{-1}}, \delta_{1}=\frac{532 b_{1} a_{0}^{4} A_{1}^{2} k_{1}^{4}}{3 A_{-1}^{2}}, q_{1}=\frac{-(-1)^{\frac{1}{4}} k_{1} a_{0} \sqrt{\frac{10 A_{1}}{3}}}{\sqrt{A_{-1}}} \\
& c_{1}=\frac{-A_{-1}^{3} d_{1}+120 a_{0}^{4} A_{1} b_{1} k_{1}^{4}}{A_{-1}^{3}}, g=\frac{-(e+h) A_{-1}^{4}-24 b_{1} a_{0}^{4} k_{1}^{4}}{A_{-1}^{4}} \\
& A_{0}=0, a_{2}=\frac{-i a_{0} A_{1}}{A_{-1}}, \delta_{1}=\frac{532 b_{1} a_{0}^{4} A_{1}^{2} k_{1}^{4}}{3 A_{-1}^{2}}, q_{1}=\frac{(-1)^{\frac{1}{4}} k_{1} a_{0} \sqrt{\frac{10 A_{1}}{3}}}{\sqrt{A_{-1}}} \\
& c_{1}=\frac{-A_{-1}^{3} d_{1}+120 a_{0}^{4} A_{1} b_{1} k_{1}^{4}}{A_{-1}^{3}}, g=\frac{-(e+h) A_{-1}^{4}-24 b_{1} a_{0}^{4} k_{1}^{4}}{A_{-1}^{4}} \\
& A_{-1}=0, A_{0}=0, \delta_{1}=-b_{1}\left(-16 a_{0}^{2} a_{2}^{2} k_{1}^{4}-12 a_{0} a_{2} k_{1}^{2} q_{1}^{2}+3 q_{1}^{4}\right), \\
& c_{1}=\frac{-A_{1}^{2} d_{1}-40 a_{0} b_{1} a_{2}^{3} k_{1}^{4}-12 a_{2}^{2} b_{1} k_{1}^{2} q_{1}^{2}}{A_{1}^{2}}, g=\frac{-(e+h) A_{-1}^{4}-24 b_{1} a_{0}^{4} k_{1}^{4}}{A_{1}^{4}} \\
& A_{1}=0, A_{0}=0, \delta_{1}=-b_{1}\left(-16 a_{0}^{2} a_{2}^{2} k_{1}^{4}-12 a_{0} a_{2} k_{1}^{2} q_{1}^{2}+3 q_{1}^{4}\right), \\
& c_{1}=\frac{-A_{-1}^{2} d_{1}-40 a_{2} b_{1} a_{0}^{3} k_{1}^{4}-12 a_{0}^{2} b_{1} k_{1}^{2} q_{1}^{2}}{A_{-1}^{2}}, g=\frac{-(e+h) A_{-1}^{4}-24 b_{1} a_{0}^{4} k_{1}^{4}}{A_{-1}^{4}} \\
& A_{1}=0, A_{0}=0, a_{2}=0, \delta_{1}=-3 b_{1} q_{1}^{4}, c_{1}=\frac{-A_{-1}^{2} d_{1}-12 b_{1} a_{0}^{2} k_{1}^{2} q_{1}^{2}}{A_{-1}^{2}}, g=\frac{-(e+h) A_{-1}^{4}-24 b_{1} a_{0}^{4} k_{1}^{4}}{A_{-1}^{4}}
\end{aligned}
$$

Now we will extract the different solutions corresponding to these 9-acceptable results, for simplicity we will choose only one result say the first one.

For the 1-st result which is,

$$
\begin{aligned}
& A_{0}=0, a_{2}=\frac{a_{0} A_{1}}{A_{-1}}, \delta_{1}=\frac{b_{1} k_{1}\left(16 a_{0}^{4} A_{1}^{2} k_{1}^{3}-24 a_{0}^{2} A_{1} A_{-1} q_{1}^{2}-3 A_{-1}^{2} q_{1}^{2}\right)}{A_{-1}^{2}}, \\
& c_{1}=\frac{-A_{-1}^{3} d_{1}+80 a_{0}^{4} A_{1} b_{1} k_{1}^{4}-12 a_{0}^{2} A_{-1} b_{1} q_{1}^{2} k_{1}^{2}}{A_{-1}^{3}}, g=\frac{-(e+h) A_{-1}^{4}-24 b_{1} a_{0}^{4} k_{1}^{4}}{A_{-1}^{4}}
\end{aligned}
$$

This result will generate 4- sub results which are,

$$
\begin{aligned}
& A_{0}=0, A_{-1}=A_{1}=1, a_{0}=a_{2}=k_{1}=q_{1}=b_{1}=c_{1}=d_{1}=\zeta_{0}=1, g+h+e=-24, \delta_{1}=-9 \\
& A_{0}=0, A_{-1}=A_{1}=1, a_{0}=a_{2}=-1, k_{1}=q_{1}=b_{1}=c_{1}=d_{1}=\zeta_{0}=1, g+h+e=-24, \delta_{1}=-9
\end{aligned}
$$




$$
\begin{aligned}
& A_{0}=0, A_{-1}=A_{1}=1, a_{0}=a_{2}=0.4, k_{1}=b_{1}=q_{1}=c_{1}=d_{1}=\zeta_{0}=1, g+h+e=-0.8, \delta_{1}=-2.3 \\
& A_{0}=0, A_{-1}=A_{1}=1, a_{0}=a_{2}=-0.4, k_{1}=b_{1}=q_{1}=c_{1}=d_{1}=\zeta_{0}=1, g+h+e=-0.8, \delta_{1}=-2.3
\end{aligned}
$$

For simplicity we will take only one of these results say the first, and extracting the corresponding solution according to the point of view of the suggested method which is:

$$
\begin{aligned}
& \varphi(\zeta)=\frac{\sqrt{a_{0} a_{2}}}{a_{2}} \tan \left(\sqrt{a_{0} a_{2}}\left(\zeta+\zeta_{0}\right), a_{0} a_{2} \mathrm{f} 0\right. \\
& \varphi(\zeta)=\tan [x-8 t+1] \\
& \psi_{1}(\zeta)=\frac{A_{-1}}{\varphi}+A_{0}+A_{1} \varphi \\
& \psi_{1}(\zeta)=\cot [x-8 t+1]+\tan [x-8 t+1] \\
& u(x, t)=\psi_{1}(\zeta) e^{i \eta_{1}(x, t)}, \zeta=k_{1} x+w_{1} t, \eta_{1}=q_{1} x+\delta_{1} t \\
& u(x, t)=(\cot [x-8 t+1]+\tan [x-8 t+1]) e^{i[x-9 t)} \\
& \operatorname{Re} u=(\cot [x-8 t+1]+\tan [x-8 t+1]) \times \cos (x-9 t) \\
& \operatorname{Im} u=(\cot [x-8 t+1]+\tan [x-8 t+1]) \times \sin (x-9 t)
\end{aligned}
$$

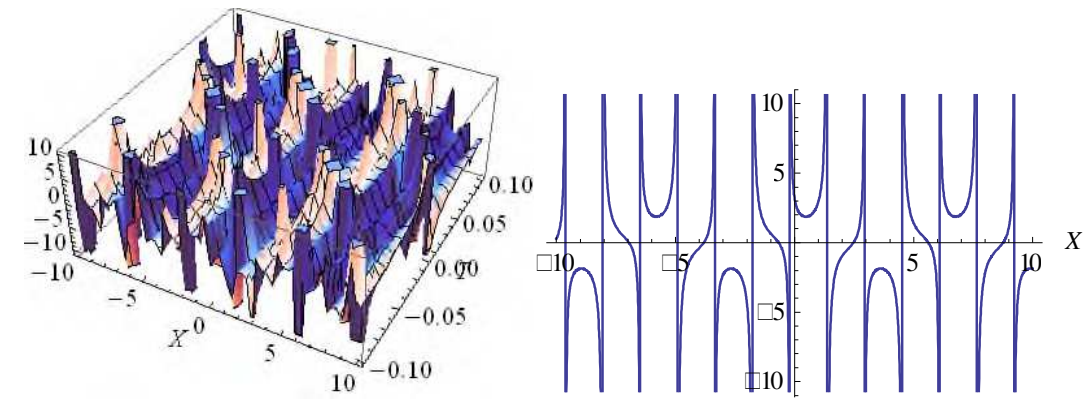

Fig.13 The cubic-quartic soliton of the Re. part of parabolic low Eq.(124) in 2D and 3D with values: $A_{0}=0, A_{-1}=A=1, a_{0}=a_{2}=k_{1}=q_{1}=b_{1}=c_{1}=d_{1}=\zeta_{0}=1, g+h+e=-24, \delta_{1}=-9, w_{1}=-8, t=0.1$ for $2 D$

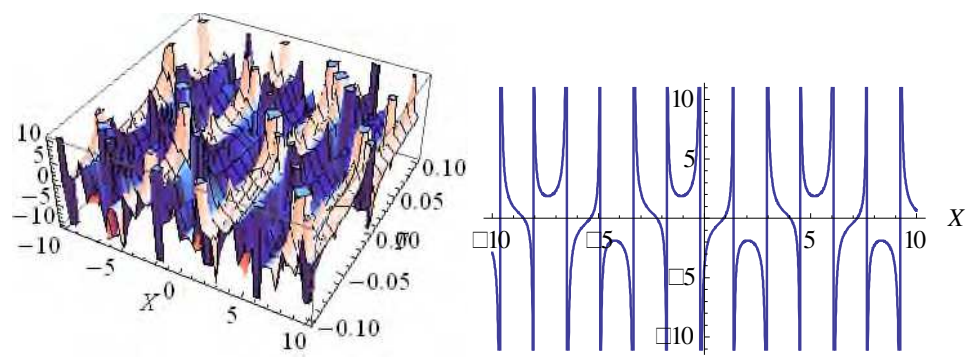

Fig.14 The cubic-quartic soliton of the Im. part of parabolic low Eq.(125) in 2D and 3D with values:

$$
A_{0}=0, A_{-1}=A_{1}=1, a_{0}=a_{2}=k_{1}=q_{1}=b_{1}=c_{1}=d_{1}=\zeta_{0}=1, g+h+e=-24, \delta_{1}=-9, w_{1}=-8, t=0.1 \text { for } 2 \mathrm{D}
$$

Via the same manner, we can plot the other solutions.

Case 2: The second family, in which $a_{0}=a_{3}=0 \Rightarrow \varphi^{\prime}=a_{1} \varphi+a_{2} \varphi^{2}$, consequently

$$
\begin{aligned}
& \psi_{1}^{\prime}=A_{1} a_{2} \varphi^{2}+a_{1} A_{1} \varphi-\frac{A_{-1} a_{1}}{\varphi}-A_{-1} a_{2} \\
& \psi_{1}^{\prime \prime}=2 A_{1} a_{2}^{2} \varphi^{3}+3 A_{1} a_{1} a_{2} \varphi^{2}+A_{1} a_{1}^{2} \varphi+A_{-1} a_{2} a_{1}+\frac{a_{1}^{2} A_{-1}}{\varphi}
\end{aligned}
$$




$$
\begin{aligned}
\psi_{1}^{\prime \prime \prime \prime}= & \frac{a_{1}^{4} A_{-1}}{\varphi}+A_{-1} a_{2} a_{1}^{3}+14 A_{1} a_{2} a_{1}^{3} \varphi^{2}+50 A_{1} a_{1}^{2} a_{2}^{2} \varphi^{3} \\
& +A_{1} a_{1}\left(1+60 a_{2}^{3}\right) \varphi^{4}+A_{1}\left(a_{1}+24 a_{2}^{4}\right) \varphi^{5}
\end{aligned}
$$

Via inserting $\psi_{1}^{\prime \prime \prime}, \psi_{1}^{\prime \prime}, \psi_{1}, \psi_{1}^{3}$ and $\psi_{1}^{5}$ into equation (106), collecting and equating the coefficients of various powers of $\varphi^{i}$ to zero leads to system of equations in which the coefficients of $\varphi^{-5}, \varphi^{-4}$ imply that $e+g+h=0$ and the coefficients of $\varphi^{-3}, \varphi^{-2}$ imply $c_{1}=-d_{1}$ and by using these new constrain at the other equations of this system it will be reduced to the following system,

$$
\begin{aligned}
& a_{1}^{4} A_{-1} b_{1} k_{1}^{4}+6 a_{1}^{2} A_{-1} b_{1} k_{1}^{2} q_{1}^{2}-3 A_{-1} b_{1} k_{1} q_{1}^{4}-A_{1} \delta_{1}=0 \\
& a_{1} A_{1} b_{1} k_{1}^{4}+24 a_{2}^{4} A_{1} b_{1} q_{1}^{4}=0 \\
& a_{1} A_{1} b_{1} k_{1}^{4}+60 a_{1} a_{2}^{3} A_{1} b_{1} q_{1}^{4}=0 \\
& 50 a_{1}^{2} a_{2}^{2} A_{1} b_{1} k_{1}^{4}+12 a_{2}^{2} A_{1} b_{1} k_{1}^{2} q_{1}^{2}=0 \\
& 14 a_{1}^{3} a_{2} A_{1} b_{1} k_{1}^{4}+18 a_{1} a_{2} A_{1} b_{1} k_{1}^{2} q_{1}^{2}=0 \\
& 6 a_{1}^{2} A_{1} b_{1} k_{1}^{2} q_{1}^{2}-3 A_{1} b_{1} q_{1}^{4}-A_{1} \delta_{1}=0 \\
& a_{1}^{3} a_{2} A_{-1} b_{1} k_{1}^{4}+6 a_{1} a_{2} A_{-1} b_{1} k_{1}^{2} q_{1}^{2}-3 A_{0} b_{1} q_{1}^{4}-A_{0} \delta_{1}=0
\end{aligned}
$$

The solution of the reduced system (129) gives the following three results,

$$
\begin{aligned}
& \text { (1) } A_{1}=0, \delta_{1}=\frac{-b_{1} k_{1}^{4} q_{1}^{4}}{12}, q_{1}=\frac{-i a_{1} k_{1}}{\sqrt{6}} \\
& \text { (2) } A_{1}=0, \delta_{1}=\frac{-b_{1} k_{1}^{4} q_{1}^{4}}{12}, q_{1}=\frac{i a_{1} k_{1}}{\sqrt{6}} \\
& \text { (3) } A_{1}=0, A_{0}=\frac{a_{2} A_{-1}}{a_{1}}, \delta_{1}=b_{1} k_{1}^{4} a_{1}^{4}+6 a_{1}^{2} b_{1} k_{1}^{2} q_{1}^{2}-3 b_{1} q_{1}^{4}
\end{aligned}
$$

We will extract the solutions in the framework of the suggested method for the first and the third result.

For the first result which is, $A_{1}=0, \delta_{1}=\frac{-b_{1} k_{1}^{4} q_{1}^{4}}{12}, q_{1}=\frac{-i a_{1} k_{1}}{\sqrt{6}}$

This result can be simplified to be,

$$
A_{0}=A_{-1}=1, A_{1}=0, a_{1}=\sqrt{6} i, a_{2}=0, k_{1}=q_{1}=d_{1}=\zeta_{0}=1, \delta_{1}=-0.1, c_{1}=-d_{1}, e+g+h=0
$$

From the point of view of the suggested method the solution is,

$$
\begin{aligned}
\varphi(\zeta) & =\frac{a_{1} \operatorname{Exp}\left[a_{1}\left(\zeta+\zeta_{0}\right)\right]}{1-a_{2} \operatorname{Exp}\left[a_{1}\left(\zeta+\zeta_{0}\right)\right]}, a_{1} \mathrm{f} 0 \\
\varphi(\zeta) & =2.45 i \operatorname{Exp}[2.45 i(x-8 t+1)] \\
\psi_{1}(\zeta) & =\frac{-i \operatorname{Exp}[-2.45 i(x-8 t+1)]}{2.45}+1 \\
\psi_{1}(\zeta) & =(1-0.4 \sin [2.45 x-19.6 t+2.45])-0.4 i \cos [2.45 x-19.6 t+2.45] \\
u(x, t) & =\psi_{1}(\zeta) e^{i \eta_{1}(x, t)}, \zeta=k_{1} x+w_{1} t, \eta_{1}=q_{1} x+\delta_{1} t
\end{aligned}
$$


$u(x, t)=(1-0.4 \sin [2.45 x-19.6 t+2.45])-0.4 i \cos [2.45 x-19.6 t+2.45] \times e^{i(x-0.1 t)}$

$\operatorname{Re} u=(1-0.4 \sin [2.45 x-19.6 t+2.45]) \cos (x-0.1 t)$

$$
+(0.4 \cos [2.45 x-19.6 t+2.45]) \sin (x-0.1 t)
$$

$\operatorname{Im} u=(1-0.4 \sin [2.45 x-19.6 t+2.45]) \sin (x-0.1 t)$

$$
-(0.4 \cos [2.45 x-19.6 t+2.45]) \cos (x-0.1 t)
$$
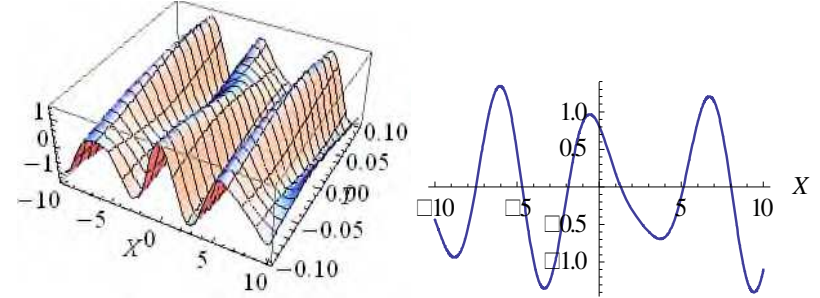

Fig.15 The cubic-quartic soliton of the Re. part of parabolic low Eq.(134) in 2D and 3D with values:

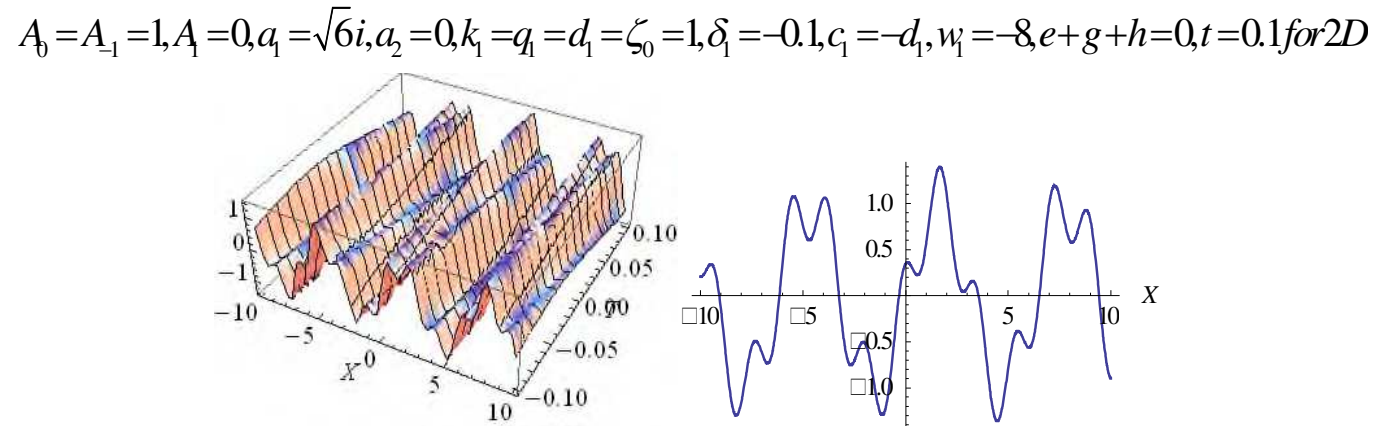

Fig.16 The cubic-quartic soliton of the Im. part of parabolic low Eq.(135) in 2D and 3D with values: $A_{0}=A_{-1}=1, A_{1}=0, a_{1}=\sqrt{6} i, a_{2}=0, k_{1}=q_{1}=d_{1}=\zeta_{0}=1, \delta_{1}=-0.1, c_{1}=-d_{1}, w_{1}=-8, e+g+h=0, t=0.1$ for $2 D$

For the third result which is, $A_{1}=0, A_{0}=\frac{a_{2} A_{-1}}{a_{1}}, \delta_{1}=b_{1} k_{1}^{4} a_{1}^{4}+6 a_{1}^{2} b_{1} k_{1}^{2} q_{1}^{2}-3 b_{1} q_{1}^{4}$

This result split into four sub results which are,

$$
\begin{aligned}
& A_{0}=A_{-1}=1, A_{1}=0, a_{1}=a_{2}=\sqrt{0.6}, k_{1}=q_{1}=d_{1}=\delta_{1}=\zeta_{0}=1, c_{1}=-d_{1}, e+g+h=0 \\
& A_{0}=A_{-1}=1, A_{1}=0, a_{1}=a_{2}=-\sqrt{0.6}, k_{1}=q_{1}=d_{1}=\delta_{1}=\zeta_{0}=1, c_{1}=-d_{1}, e+g+h=0 \\
& A_{0}=A_{-1}=1, A_{1}=0, a_{1}=a_{2}=i \sqrt{6.6}, k_{1}=q_{1}=d_{1}=\delta_{1}=\zeta_{0}=1, c_{1}=-d_{1}, e+g+h=0 \\
& A_{0}=A_{-1}=1, A_{1}=0, a_{1}=a_{2}=-i \sqrt{6.6}, k_{1}=q_{1}=d_{1}=\delta_{1}=\zeta_{0}=1, c_{1}=-d_{1}, e+g+h=0
\end{aligned}
$$

We will plot only one say the first, from the point of view of the suggested method the solution is,

$$
\begin{aligned}
\varphi(\zeta) & =\frac{a_{1} \operatorname{Exp}\left[a_{1}\left(\zeta+\zeta_{0}\right)\right]}{1-a_{2} \operatorname{Exp}\left[a_{1}\left(\zeta+\zeta_{0}\right)\right]}, a_{1} \mathrm{f} 0 \\
\varphi(\zeta) & =\frac{0.8 \operatorname{Exp}[0.8 x-6.4 t+0.8]}{1-0.8 \operatorname{Exp}[0.8 x-6.4 t+0.8]} \\
\psi_{1}(\zeta) & =1.3 \operatorname{Exp}[-0.8 x+6.4 t-0.8]-1 \\
u(x, t) & =(1.3 \operatorname{Exp}[-0.8 x+6.4 t-0.8]-1) \times e^{i(x+t)} \\
\operatorname{Re} u & =(1.3 \operatorname{Exp}[-0.8 x+6.4 t-0.8]-1) \times \cos (x+t) \\
\operatorname{Im} u & =(1.3 \operatorname{Exp}[-0.8 x+6.4 t-0.8]-1) \times \sin (x+t)
\end{aligned}
$$




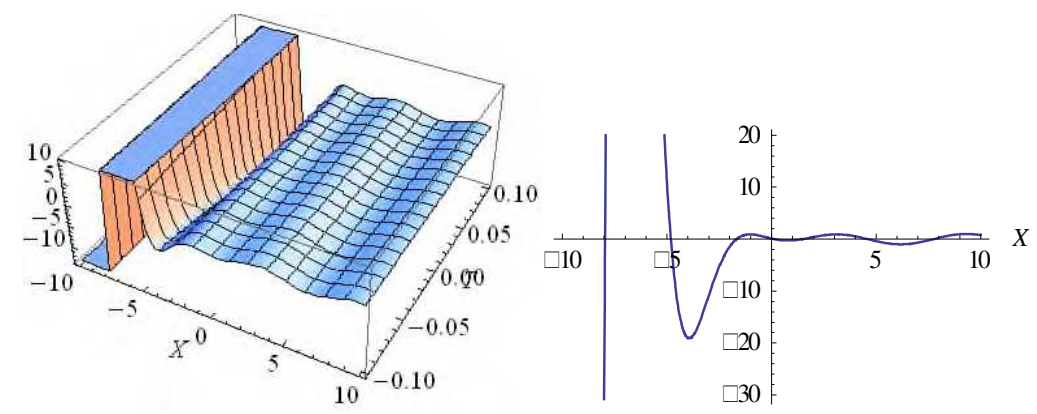

Fig.17 The cubic-quartic soliton of the Re. part of parabolic low Eq.(139) in 2D and 3D with values: $A_{0}=A_{-1}=1, A_{1}=0, a_{1}=a_{2}=0.8, k_{1}=q_{1}=d_{1}=\zeta_{0}=\delta_{1}=1, c_{1}=-d_{1}, w_{1}=-8, e+g+h=0, t=0.1$ for $2 D$

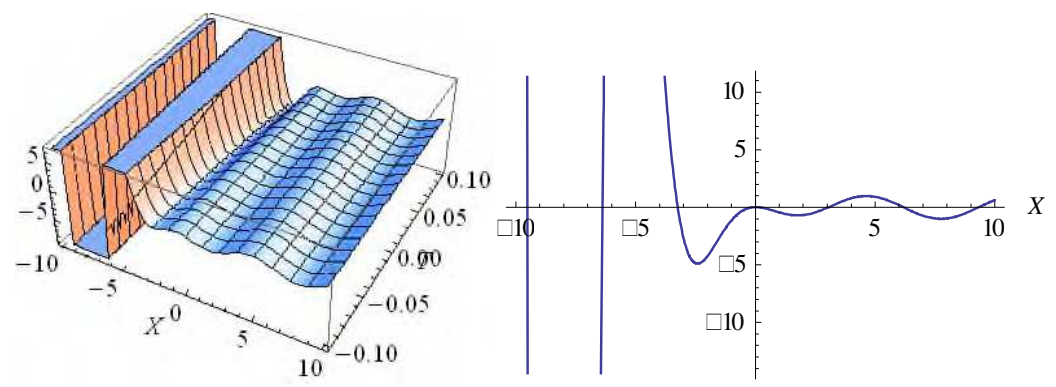

Fig.18 The cubic-quartic soliton of the Im. part of parabolic low Eq.(140) in 2D and 3D with values: $A_{0}=A_{-1}=1, A_{1}=0, a_{1}=a_{2}=0.8, k_{1}=q_{1}=d_{1}=\zeta_{0}=\delta_{1}=1, c_{1}=-d_{1}, w_{1}=-8, e+g+h=0, t=0.1$ for $2 \mathrm{D}$

By the same manner we can plot the other remained cases.

\section{The Cubic-Quartic NLSE for the Non-Local Low nonlinearity using the ESEM}

The cubic-quartic NLSE in polarization-preserving fibers with non-local low be proposed in the form,

$$
i Q_{t}+i a Q_{x x x}+b Q_{x x x x}+\left(c\left|Q^{2}\right|\right)_{x x} Q=0
$$

The cubic-quartic NLSE in birefringent fiber non-local low is,

$$
\begin{array}{r}
i u_{t}+i a_{1} u_{x x x}+b_{1} u_{x x x x}+\left(c_{1}\left(|u|^{2}\right)_{x x}+d_{1}\left(|v|^{2}\right)_{x x}\right) u=0 \\
i v_{t}+i a_{2} v_{x x x}+b_{1} v_{x x x x}+\left(c_{2}\left(|v|^{2}\right)_{x x}+d_{2}\left(|u|^{2}\right)_{x x}\right) v=0
\end{array}
$$

Via inserting the relations (13-26) into equation (142) and (143) will transform them to the following real and imaginary equations respectively:

$$
\begin{gathered}
\operatorname{Re} \Rightarrow b_{j} k_{j}^{4} \psi_{j}^{\prime \prime \prime}-\left(3 a_{j} q_{j} k_{j}^{2}+6 b_{j} k_{j}^{2} q_{j}^{2}\right) \psi_{j}^{\prime \prime}+\left(b_{j} q_{j}^{4}+a_{j} q_{j}^{3}-\delta_{j}\right) \psi_{j}+\left(2 c_{j}+2 d_{j}\right) \psi_{j} \psi_{j}^{2}+\left(2 c_{j}+2 d_{j}\right) \psi_{j}^{\prime \prime} \psi_{j}^{2}=0 \\
\operatorname{Im} \Rightarrow k_{j}^{3}\left(a_{j}+4 b_{j} q_{j}\right) \psi_{j}^{\prime \prime \prime}+\left(w_{j}-3 a_{j} k_{j} q_{j}^{2}-4 b_{j} k_{j} q_{j}^{3}\right) \psi_{j}^{\prime}=0
\end{gathered}
$$

Equation (145) implies $a_{j}=-4 b_{j} q_{j}, w_{j}=3 k_{j} a_{j} q_{j}^{2}+4 k_{j} b_{j} q_{j}^{3}$, hence under this constrain Eq. (144) will be converted to,

$$
b_{j} k_{j}^{4} \psi_{j}^{\prime \prime \prime \prime}+6 b_{j} k_{j}^{2} q_{j}^{2} \psi_{j}^{\prime \prime}-\left(3 b_{j} q_{j}^{4}+\delta_{j}\right) \psi_{j}+\left(2 c_{j}+2 d_{j}\right) \psi_{j} \psi_{j}^{\prime 2}+\left(2 c_{j}+2 d_{j}\right) \psi_{j}^{\prime \prime} \psi_{j}^{2}=0
$$

Equation (146) represents either the first part Eq. (142) or the second part Eq (143) which is the same, for similarity we will implement the solution for the first part $j=1$ which is,

$$
b_{1} k_{1}^{4} \psi_{1}^{\prime \prime \prime}+6 b_{1} k_{1}^{2} q_{1}^{2} \psi_{1}^{\prime \prime}-\left(3 b_{1} q_{1}^{4}+\delta_{1}\right) \psi_{1}+\left(2 c_{1}+2 d_{1}\right) \psi_{1} \psi_{1}^{2}+\left(2 c_{1}+2 d_{1}\right) \psi_{1}^{\prime \prime} \psi_{1}^{2}=0
$$


Via balancing $\psi_{j}^{\prime \prime \prime \prime}, \psi^{\prime \prime} \psi_{j}^{2}$ at Eq. (147) leads to $4 N=2 N+N+2$ which implies that $N=2$, hence the solution is,

$$
\psi_{1}(\zeta)=\frac{A_{-2}}{\varphi^{2}}+\frac{A_{-1}}{\varphi}+A_{0}+A_{1} \varphi+A_{2} \varphi^{2}
$$

Where $\varphi^{\prime}=a_{0}+a_{1} \varphi+a_{2} \varphi^{2}+a_{3} \varphi^{3}$

Case 1: The first family, in which $a_{1}=a_{3}=0 \Rightarrow \varphi^{\prime}=a_{0}+a_{2} \varphi^{2}$, consequently

$$
\begin{aligned}
\psi_{1}^{\prime}= & \frac{-2 a_{0} A_{-2}}{\varphi^{3}}-\frac{a_{0} A_{-1}}{\varphi^{2}}-\frac{2 a_{2} A_{-2}}{\varphi}+\left(A_{1} a_{0}-a_{2} A_{-1}\right)+2 A_{2} a_{0} \varphi+\left(A_{1} a_{2}+2 a_{2} A_{2}\right) \varphi^{2} . \\
\psi_{1}^{\prime \prime}= & \frac{6 a_{0}^{2} A_{-2}}{\varphi^{4}}+\frac{2 a_{0}^{2} A_{-1}}{\varphi^{3}}+\frac{8 a_{0} a_{2} A_{-2}}{\varphi^{2}}+\frac{2 a_{0} a_{2} A_{-1}}{\varphi}+2\left(A_{2} a_{0}^{2}+A_{-2} a_{2}^{2}\right) \\
& 2 a_{0} a_{2} A_{1} \varphi++8 a_{0} a_{2} A_{2} \varphi^{2}+2 a_{2}^{2} A_{1} \varphi^{3}+6 a_{2}^{2} A_{2} \varphi^{4} . \\
\psi_{1}^{\prime \prime \prime}= & \frac{120 a_{0}^{4} A_{-2}}{\varphi^{6}}+\frac{24 a_{0}^{4} A_{-1}}{\varphi^{5}}+\frac{240 a_{0}^{3} a_{2} A_{-2}}{\varphi^{4}}+\frac{40 a_{0}^{3} a_{2} A_{-1}}{\varphi^{3}} \\
+ & \frac{136 a_{0}^{2} a_{2}^{2} A_{-2}}{\varphi^{2}}+\frac{16 a_{0}^{2} a_{2}^{2} A_{-1}}{\varphi}+16 a_{0} a_{2}^{3} A_{-2}+16 a_{0}^{3} a_{2} A_{2}+16 a_{0}^{2} a_{2}^{2} A_{1} \varphi \\
+ & 136 a_{0}^{2} a_{2}^{2} A_{2} \varphi^{2}+40 a_{0} a_{2}^{3} A_{1} \varphi^{3}+240 a_{0} a_{2}^{3} A_{2} \varphi^{4}+24 a_{2}^{4} A_{1} \varphi^{5}+120 a_{2}^{4} A_{2} \varphi^{6}
\end{aligned}
$$

Via inserting $\psi_{1}^{\prime \prime \prime \prime}, \psi_{1}^{2} \psi_{1}^{\prime \prime}, \psi_{1}^{\prime \prime}, \psi_{1} \psi_{1}^{\prime 2}$ and $\psi_{1}^{3}$ into equation (147), collecting and equating the coefficients of various powers of $\varphi^{i}$ to zero leads to system of equations, by solving this system rejected results will be achieved because in all these results either $a_{0}=0$ or $a_{2}=0$ or $A_{-2}=A_{-1}=A_{1}=A_{2}=0$, hence all these results will be refused.

Case 2: The second family, in which $a_{0}=a_{3}=0 \Rightarrow \varphi^{\prime}=a_{1} \varphi+a_{2} \varphi^{2}$, consequently

$$
\begin{gathered}
\psi_{1}^{\prime}=A_{1} a_{2} \varphi^{2}+a_{1} A_{1} \varphi-\frac{A_{-1} a_{1}}{\varphi}-A_{-1} a_{2} \\
\psi_{1}^{\prime \prime}=2 A_{1} a_{2}^{2} \varphi^{3}+3 A_{1} a_{1} a_{2} \varphi^{2}+A_{1} a_{1}^{2} \varphi+A_{-1} a_{2} a_{1}+\frac{a_{1}^{2} A_{-1}}{\varphi} \\
\psi_{1}^{\prime \prime \prime \prime}=\frac{a_{1}^{4} A_{-1}}{\varphi}+A_{-1} a_{2} a_{1}^{3}+14 A_{1} a_{2} a_{1}^{3} \varphi^{2}+50 A_{1} a_{1}^{2} a_{2}^{2} \varphi^{3} \\
+A_{1} a_{1}\left(1+60 a_{2}^{3}\right) \varphi^{4}+A_{1}\left(a_{1}+24 a_{2}^{4}\right) \varphi^{5}
\end{gathered}
$$

Via inserting $\psi_{1}^{\prime \prime \prime}, \psi_{1}^{2} \psi_{1}^{\prime \prime}, \psi_{1}^{\prime \prime}, \psi_{1} \psi_{1}^{\prime 2}$ and $\psi_{1}^{3}$ into equation (147), collecting and equating the coefficients of various powers of $\varphi^{i}$ zero, will lead to a system of equations, by solving this system only six acceptable solutions are achieved and the remain are refused because either $a_{1}=0$ for which there are no solution according to the suggested method or $A_{-2}=A_{-1}=A_{1}=A_{2}=0$ or $b_{1}=0$.

The acceptable results are, 


$$
\begin{aligned}
& \text { (1) } A_{-1}=A_{1}=A_{2}=0, a_{2}^{2}=\frac{-5 A_{0}\left(3 b_{1} q_{1}^{4}+\delta_{1}\right)}{108 A_{-2} b_{1} k_{1}^{2} q_{1}^{2}}, a_{1}=-1.1 i \frac{q_{1}}{k_{1}}, c_{1}=-d_{1} \\
& \text { (2) } A_{-1}=A_{1}=A_{2}=0, a_{2}^{2}=\frac{-5 A_{0}\left(3 b_{1} q_{1}^{4}+\delta_{1}\right)}{108 A_{-2} b_{1} k_{1}^{2} q_{1}^{2}}, a_{1}=1.1 i \frac{q_{1}}{k_{1}}, c_{1}=-d_{1} \\
& \text { (3) } A_{-1}=A_{1}=A_{2}=a_{2}=0, a_{1}=-1.1 i \frac{q_{1}}{k_{1}}, b_{1}=\frac{-\delta_{1}}{3 q_{1}^{4}}, c_{1}=-d_{1} \\
& \text { (4) } A_{-1}=A_{1}=A_{2}=a_{2}=0, a_{1}=1.1 i \frac{q_{1}}{k_{1}}, b_{1}=\frac{-\delta_{1}}{3 q_{1}^{4}}, c_{1}=-d_{1} \\
& \text { (5) } A_{-2}=A_{2}=a_{2}=0, A_{-1}=0.3 A_{1}, a_{1}=-1.1 i \frac{q_{1}}{k_{1}}, c_{1}=-d_{1} \\
& \text { (6) } A_{-2}=A_{2}=a_{2}=0, A_{-1}=0.3 A_{1}, a_{1}=1.1 i \frac{q_{1}}{k_{1}}, c_{1}=-d_{1}
\end{aligned}
$$

For simplicity and repetition we will establish the solutions corresponding to the first and the six results and plot them.

For the first result which is,

$$
A_{-2}=A_{2}=a_{2}=0, A_{-1}=0.3 A_{1}, a_{1}=1.1, c_{1}=-d_{1}
$$

This result can be simplified to be,

$$
A_{-1}=A_{1}=A_{2}=0,\left|a_{1}\right|=1.1,\left|a_{2}\right|=0.44, A_{0}=A_{-2}=q_{1}=k_{1}=\delta_{1}=1, c_{1}=-d_{1}
$$

The solution in the framework of the suggested method is,

$$
\psi_{1}(\zeta)=\frac{A_{-2}}{\varphi^{2}}+\frac{A_{-1}}{\varphi}+A_{0}+A_{1} \varphi+A_{2} \varphi^{2}
$$

Where,

$$
\begin{aligned}
& \varphi(\zeta)=\frac{1.1 \operatorname{Exp}\left[1.1\left(\zeta+\zeta_{0}\right)\right]}{1-0.44 \operatorname{Exp}\left[1.1\left(\zeta+\zeta_{0}\right)\right]} \\
& \psi_{1}(x, t)=0.8 \operatorname{Exp}\left[-2.2\left(\zeta+\zeta_{0}\right)\right]-0.7 \operatorname{Exp}\left[-1.1\left(\zeta+\zeta_{0}\right)\right]+1.2 \\
& \psi_{1}(x, t)=\left(1.2+0.8 \operatorname{Exp}\left[-2.2\left(\zeta+\zeta_{0}\right)\right]-0.7 \operatorname{Exp}\left[-1.1\left(\zeta+\zeta_{0}\right)\right]\right) \times e^{i(x+t)} \\
& \operatorname{Re} \psi_{1}(x, t)=\left(1.2+0.8 \operatorname{Exp}\left[-2.2\left(\zeta+\zeta_{0}\right)\right]-0.7 \operatorname{Exp}\left[-1.1\left(\zeta+\zeta_{0}\right)\right]\right) \times \cos (x+t) \\
& \operatorname{Im} \psi_{1}(x, t)=\left(1.2+0.8 \operatorname{Exp}\left[-2.2\left(\zeta+\zeta_{0}\right)\right]-0.7 \operatorname{Exp}\left[-1.1\left(\zeta+\zeta_{0}\right)\right]\right) \times \sin (x+t)
\end{aligned}
$$

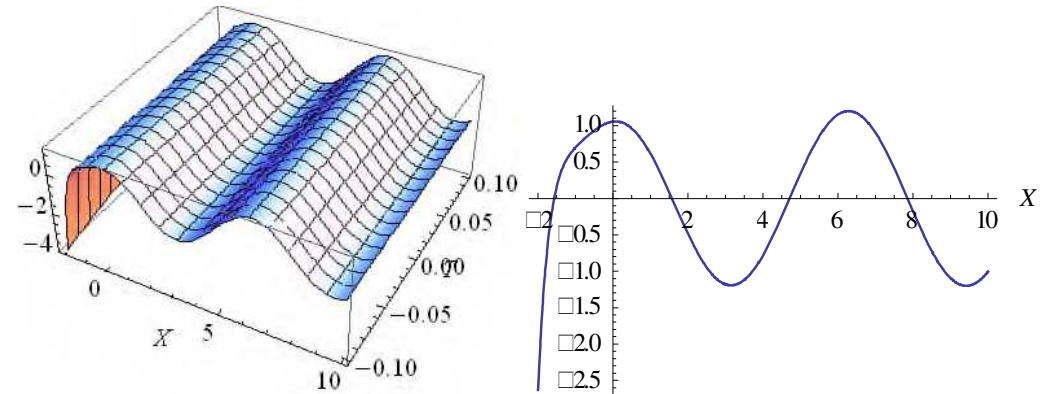

Fig.19 The cubic-quartic soliton of the Re. part of non-local low Eq.(160) in 2D and 3D with values:

$$
A_{-1}=A_{1}=A_{2}=0,\left|a_{1}\right|=1.1,\left|a_{2}\right|=0.44, A_{0}=A_{-2}=q_{1}=k_{1}=\delta_{1}=1, c_{1}=-d_{1}
$$




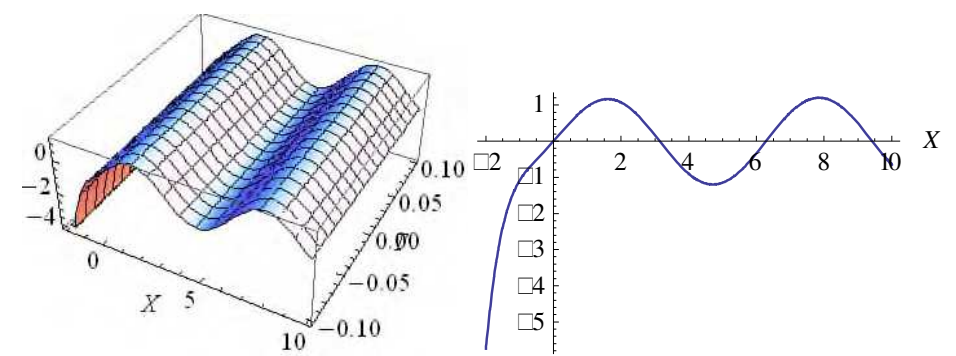

Fig.20 The cubic-quartic soliton of the Im. part of non-local low Eq.(161) in 2D and 3D with values:

$$
A_{-1}=A_{1}=A_{2}=0,\left|a_{1}\right|=1.1,\left|a_{2}\right|=0.44, A_{0}=A_{2}=q_{1}=k_{1}=\delta_{1}=1, c_{1}=-d_{1}
$$

For the six result which is,

$$
A_{-1}=A_{1}=A_{2}=0, a_{2}^{2}=\frac{-5 A_{0}\left(3 b_{1} q_{1}^{4}+\delta_{1}\right)}{108 A_{-2} b_{1} k_{1}^{2} q_{1}^{2}}, a_{1}=-1.1 i \frac{q_{1}}{k_{1}}, c_{1}=-d_{1}
$$

This result can be simplified to be,

$$
A_{-2}=A_{0}=A_{2}=a_{2}=0, A_{-1}=0.3,\left|a_{1}\right|=1.1, A_{1}=q_{1}=k_{1}=\delta_{1}=1, c_{1}=-d_{1}
$$

The solution in the framework of the suggested method is,

$$
\begin{aligned}
& \varphi(\zeta)=1.1 \operatorname{Exp}\left[1.1\left(\zeta+\zeta_{0}\right)\right] \\
& \psi_{1}(\zeta)=0.3 \operatorname{Exp}\left[-1.1\left(\zeta+\zeta_{0}\right)\right]+1.1 \operatorname{Exp}\left[1.1\left(\zeta+\zeta_{0}\right)\right] \\
& \psi_{1}(x, t)=\left(0.3 \operatorname{Exp}\left[-1.1\left(\zeta+\zeta_{0}\right)\right]+1.1 \operatorname{Exp}\left[1.1\left(\zeta+\zeta_{0}\right)\right]\right) \times e^{i(x+t)} \\
& \operatorname{Re} \psi_{1}(x, t)=\left(0.3 \operatorname{Exp}\left[-1.1\left(\zeta+\zeta_{0}\right)\right]+1.1 \operatorname{Exp}\left[1.1\left(\zeta+\zeta_{0}\right)\right]\right) \times \cos (x+t) \\
& \operatorname{Im} \psi_{1}(x, t)=\left(0.3 \operatorname{Exp}\left[-1.1\left(\zeta+\zeta_{0}\right)\right]+1.1 \operatorname{Exp}\left[1.1\left(\zeta+\zeta_{0}\right)\right]\right) \times \sin (x+t)
\end{aligned}
$$
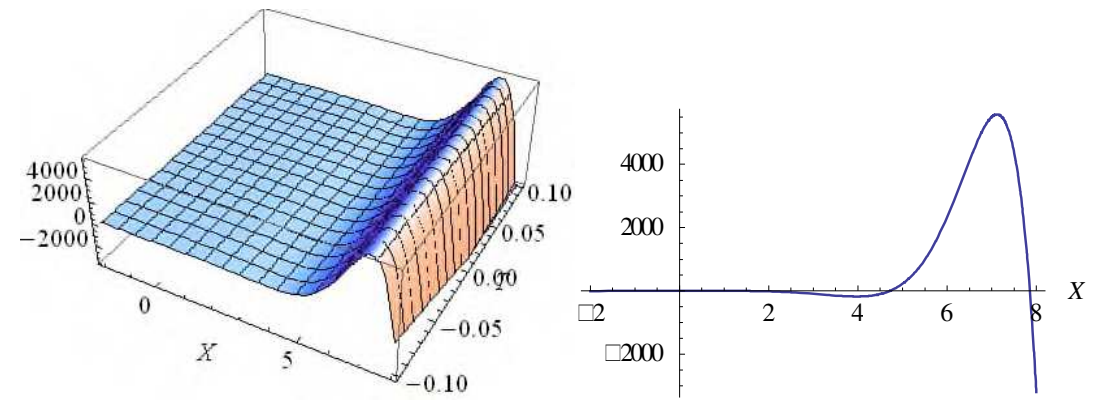

Fig.21 The cubic-quartic soliton of the Re. part of non-local low Eq.(160) in 2D and 3D with values:

$$
A_{-2}=A_{0}=A_{2}=a_{2}=0, A_{-1}=0.3,\left|a_{1}\right|=1.1, A_{1}=q_{1}=k_{1}=\delta_{1}=1, c_{1}=-d_{1}
$$
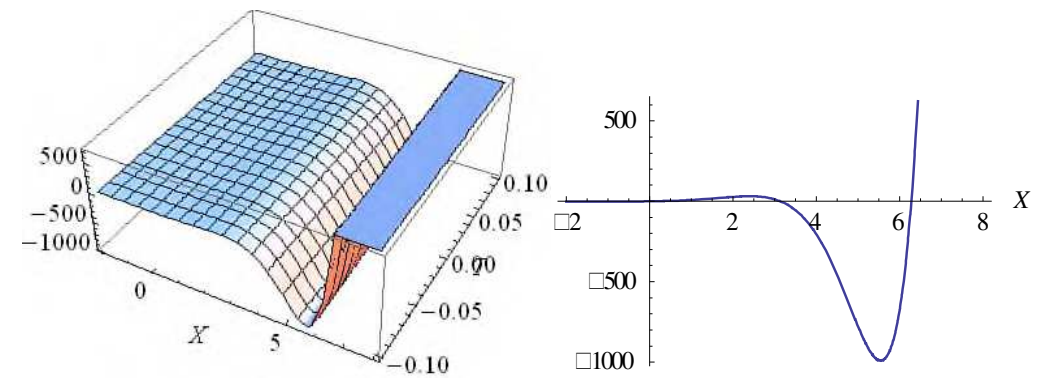

Fig.22 The cubic-quartic soliton of the Im. part of non-local low Eq.(161) in 2D and 3D with values:

$$
A_{-2}=A_{0}=A_{2}=a_{2}=0, A_{-1}=0.3,\left|a_{1}\right|=1.1, A_{1}=q_{1}=k_{1}=\delta_{1}=1, c_{1}=-d_{1}
$$

By the same manner we can plot the other results. 


\section{9-Conclusion}

In this paper, multiple accurate types of solitons to the cubic-quartic NLSE in polarization-preserving fibers with four various forms are implemented via the ESEM. Firstly, through overview Fig. (1-10) it is clear ability of the suggested method to give good description of the new types of solitons to the cubic-quartic NLSE for the Kerr-Low NLSE in polarization-preserving fibers. Also, via overview Fig.(11-12) we are able to document exact types of solitons to the cubic-quartic NLSE for the Quadratic-Low in polarization-preserving fibers coupled equations by the same method. In related subject, the ESEM has been used effectively to establish new multiple accurate types of solitons to the cubic-quartic NLSE for the Parabolic-Low NLSE in polarization-preserving fibers coupled equations which appear clearly in Fig. (13-18). Finally, new types of solitons to the cubic-quartic NLSE for the Nonlocal Low in polarization-preserving fibers were achieved in the framework of the same method Fig (19-22). The obtained multiple new solitons which weren't achieved before by any other authors for the four forms mentioned before denote to the novelty of these results, especially compared with that achieved previously by [25-29] who used various manners to study these forms that emerged from this model. Consequently, new distinct and impressive visions of the solitons to the quartic-cubic NLSE for birefringent fibers of these four different forms have been constructed and the achieved solitons will add improved extended studies for all modern telecommunication engineering and all related phenomena.

\section{Ethics Declarations}

Conflict of interest: The authors declare that they have no conflict of interest.

Availability of Data and Material: Not available.

Code availability : Not available.

\section{References}

[1] Erasmus Bartholin, Experimenta crystalli islandici disdiaclastici quibus mira \& infolita refractio detegitur [Experiments on birefringent Icelandic crystal through which is detected a remarkable and unique refraction] (Copenhagen, Denmark: Daniel Paulli, 1669).

[2] Neves, N. M. (1998). "The use of birefringence for predicting the stiffness of injection molded polycarbonate discs". Polymer Engineering \& Science. 38 (10): 1770-1777.

[3] Brad Amos. Birefringence for factors I: what is birefringence? Archived December 14, 2013, at the Wayback Machine First published in StoneChat, the Journal of the UK Facet Cutter's Guild. January-March. edition 2005.

[4] Nofal, T.A., Simple equation method for nonlinear partial differential equations and its applications, J. Egypt. Math. Soc. 24 (2016) 204-209.

[5] Kudryashov, N.A., (2020); Mathematical model of propagation pulse in optical fiber with power nonlinearities, Optik 212, 164750.

[6] Kudryashov, N.A.,(2020); Highly dispersive solitary wave solutions of perturbed nonlinear Schrödinger equations, Appl. Math. Comput. 371; 124972.

[7] Kudryashov, N.A., (2020); Highly dispersive optical solitons of the generalized nonlinear eighth-order Schrödinger equation, Optik 206, 164335.

[8] Li, B., Zhao, J., Pan, A., Mirzazadeh, M., Ekici, M., Zhou, Q., Liu, W., (2019); Stable propagation of optical solitons in fiber lasers by using symbolic computation, Optik 178, 142 145.

[9] Liu, C., (2005); Exact solutions for the higher-order nonlinear Schrodinger equation in nonlinear optical fibres. Chaos, Solitons Fractals 23, 949-955. 
[10] Xiao, L.L., Liang, W.M., (2009); The $\left(\mathrm{G}^{\prime} / \mathrm{G}\right)$-Expansion Method and travelling wave solutions for a higher-order nonlinear Schrodinger equation, Appl. Math. Comput. 208, 440445.

[11] El-Ganaini, S.I.A., (2013); The first integral method to the nonlinear Schrodinger equations in higher dimensions, Abst. Appl. Anal., 10 ID349173.

[12] Mirzazadeh, M., Eslami, M., and Biswas, A., (2014); Dispersive optical solitons by Kudryashov's method; Optik, 125, 23, 6874-6880

[13] Shehata, M.S.M., Rezazadeh, H., Jawad, A.J.M., Zahran, E.H.M., and Bekir, A., (2020); Optical solitons to a perturbed Gerdjikov-Ivanov equation using two different techniques, Revista Mexicana de Física, in press

[14] Bekir,A., Zahran, E.H.M., (2021); New visions of the soliton solutions to the modified nonlinear Schrodinger equation; Optik, in press.

[15] Biswas, A., Ullah, M.Z., Asma, M., Zhou, Q., Moshokoa, S.P., Belic, B., (2017); Optical solitons with quadratic-cubic nonlinearity by semi-inverse variational principle, Optik 139, $16-19$.

[16] Biswas, A., Triki, H., Zhou, Q., Moshokoa, S.P., Ullah, M.Z., Belic, M., (2017); Cubicquartic optical solitons in Kerr and power law media, Optik 144; 357-362.

[17] Biswas, A., Kara, A.H., Ullah, M.Z., Zhou, Q., Triki, H., Belic, M., (2017); Conservation laws for cubic-quartic optical solitons in Kerr and power law media, Optiki 145, 650-654.

[18] Bansal,A., Biswas, A., Zhou, Q. , Babatin, M.M., (2018); Lie symmetry analysis for cubic-quartic nonlinear Schrödinger's equation, Optik 169;12-15.

[19] Biswas, A., Arshed, S., (2018); Application of semi-inverse variational principle to cubic-quartic optical solitons with Kerr and power law nonlinearity, Optik 172; 847-850.

[20] Das, A., Biswas, A., Ekici, M., Khan, S., Zhou, Q., Moshokoa, S.P., (2019); Suppressing internet bottleneck with fractional temporal evolution of cubic-quartic optical solitons, Optik 182; 303-307.

[21] Gonzalez-Gaxiola, O., Biswas, A., Mallawi, F., Belic, M., (2020); Cubic-quartic bright optical solitons by improved Adomian decomposition method, J. Adv. Res. 21, 161-167.

[22] Kohl, R.W., Biswas, A., Ekici, M., Zhou, Q., Moshokoa, S.P., Belic, M.R., (2019); Cubic-quartic optical soliton perturbation by semi-inverse variational principle, Optik 185, 45-49.

[23] Bekir, A., Zahran, E.H.M., (2020); Bright and dark soliton solutions for the complex Kundu-Eckhaus equation; Optik; 223, 165233

[24] Bekir, A., Zahran, E., (2021); Three distinct and impressive visions for the soliton solutions to the higher-order nonlinear Schrodinger equation; Optik, 228; 166157

[25] Kudryashov, N.A., Loguinova, N.B., (2008); Extended simplest equation method for nonlinear differential equations, Appl. Math. Comput. 205; 396-402

[26] Wazwaz, A.M., and Xu, G.Q., (2020); Bright, dark, and Gaussons optical solutions for fourth order Schrodinger equation with cubic-quantic and logarithmic nonlinearities, Optik; $202 ; 163564$.

[27] Yildirim, Y., Biswas, A., Guggilla, P., Mallawi, F., Belic, M.R., (2020); Cubic-quartic optical solitons in birefringent fibers with four forms of nonlinear refractive index, Optik 203, 163885.

[28] Yildirim, Y., Biswas, A., Jawad, A.J.M., Ekici, M., Zhou, Q., Khan, S., Alzahrani, A.K., and Belic, M.R., (2020); Cubic-quartic optical solitons in birefringent fibers with four forms of nonlinear refractive index by exp-function expansion, Results Phys.16, 102913. 